\title{
Modeling Time and Valuation in Structured Argumentation Frameworks
}

\author{
Maximiliano C. D. Budán ${ }^{\mathrm{a}, \mathrm{b}, \mathrm{c}, *}$, Mauro Gómez Lucero $^{\mathrm{a}}$, Carlos Chesñevara ${ }^{\mathrm{a}, \mathrm{b}}$, \\ Guillermo R. Simari ${ }^{\mathrm{a}}$ \\ ${ }^{a}$ Artificial Intelligence Research and Development Laboratory - Department of Computer \\ Science and Engineering, Universidad Nacional del Sur, Argentina \\ ${ }^{b}$ Departament of Mathematics, Universidad Nacional de Santiago del Estero, Argentina \\ ${ }^{c}$ Consejo Nacional de Investigaciones Científicas y Técnicas, Argentina \\ Phone: (+54)(291)-4595135 / Fax: (+54)(291)-4595136
}

\begin{abstract}
Temporal Argumentation Frameworks (TAF) represent a recent extension of Dung's abstract argumentation frameworks that consider the temporal availability of arguments. In a $T A F$, arguments are valid during specific time intervals, called availability intervals, while the attack relation of the framework remains static and permanent in time; thus, in general, when identifying the set of acceptable arguments, the outcome associated with a $T A F$ will vary in time. We introduce an extension of $T A F$, called Extended Temporal Argumentation Framework $(E-T A F)$, adding the capability of modeling the temporal availability of attacks among arguments, thus modeling special features of arguments varying over time and the possibility that attacks are only available in a given time interval.

E-TAF will be enriched by considering Structured Abstract Argumentation, using Dynamic Argumentation Frameworks. The resulting framework, $E$ - $T A F^{*}$, provides a suitable model for different time-dependent issues satisfying properties and equivalence results that permit to contrast the expressivity of $E$-TAF and $E-T A F^{*}$ with argumentation based on abstract frameworks. Thus, the main contribution here is to provide an enhanced framework for modeling special features of argumentation varying over time, which are relevant in many real-world situations. The proposal aims at advancing in the integration of time and valuation in the context of argumentation systems as well.
\end{abstract}

Keywords: Argumentation, Temporal availability, Time-dependent Issues.

\footnotetext{
* Corresponding author

Email addresses: mcdb@cs.uns.edu.ar (Maximiliano C. D. Budán), mjg@cs.uns.edu.ar (Mauro Gómez Lucero), cic@cs.uns.edu.ar (Carlos Chesñevar), grs@cs.uns.edu.ar (Guillermo R. Simari)
} 


\section{Introduction}

Human commonsense reasoning is in many occasions the result of an analysis of alternatives and the evaluation of their support. The study of this process suggested several formalisms that were introduced attempting to provide a way of modeling this useful mechanism; notably, the field of argumentation has contributed with many proposals since ancient times [12, 14, 39, 35]. Thus, argumentation can be associated with the interaction of arguments for and against a claim supported by some form of reasoning from a set of premises, with the purpose of ascertaining if that conclusion is acceptable.

A variety of argument-based formalisms have emerged providing useful tools in areas such as legal reasoning, autonomous agents, and multi-agent systems [30, $38,14,5,40,36,44,11]$. In such environments, an agent may use argumentation to perform individual reasoning to reach a resolution over contradictory evidence or to decide between conflicting goals; but also, multiple agents may use dialectical argumentation to identify and settle differences, interacting via different processes such as negotiation, persuasion, or joint deliberation. Many of such accounts of argumentation are based on Dung's foundational work characterizing Abstract Argumentation Frameworks [24] where arguments are considered as atomic entities and their interaction is represented solely through an attack relation. In an effort to extend the knowledge representation abilities of the system, other research approaches have considered different possibilities of representation of the internal structure of the arguments [13, 29, 26, 37].

In many cases, commonsense reasoning requires the representation of time; thus, its consideration is also of relevance in the modeling of the argumentation capabilities of intelligent agents $[6,7,33]$. Temporal Argumentation Frameworks $(T A F)[22,23]$ are a recent extension of Dung's abstract frameworks that consider the temporal availability of arguments. In a $T A F$, arguments are valid only during specific time intervals that are referred to as availability intervals; thus, when identifying the set of acceptable arguments the outcome associated with a $T A F$ will vary in time. Although arguments in TAF become associated with availability intervals, the attack relation between them is assumed to be static and permanent in time, i.e., if an argument is available at a given time any attack in which it participates effectively occurs.

In what follows, we will develop two formalisms starting from existing ones. We will take as point of departure the Temporal Argumentation Frameworks, and then we will trim down the formalism of Dynamic Argumentation Frameworks to a simpler framework; later, these two formalisms will be put together as a novel approach. We will briefly describe these two changes below.

In Extended Temporal Argumentation Frameworks (E-TAF) we will add to TAFs the capability of modeling the availability of attacks between arguments. This novel feature of $E-T A F$ will allow to model special features of arguments varying over time, where an attack can be only available in a given time interval with the intended meaning that the attacking argument is more reliable than the attacked one in this interval. 
The formalism of Structured Abstract Argumentation (SAA) is based on a simplified version of the recently introduced Dynamic Argumentation Frameworks [41]. One of the technical contributions in their design is to consider structural elements for the arguments involved, thus expanding the representation capabilities of Dung's frameworks but maintaining a degree of abstraction. In this formalization, arguments are represented as structures standing for trees of smaller abstract entities corresponding to individual reasoning steps. These individual reasoning steps may be considered as abstractions of rules in a rule-based argumentation system without having to the make explicit use of a concrete knowledge representation language. Using this type of frameworks with less abstract characteristics, we can enrich the $E-T A F$, and the resulting framework $E-T A F^{*}$ will be able to provide a suitable model for different timedependent issues, such as reliability, strength, or skills; thus, these features can be associated with arguments to facilitate the building of applications in several real-world situations.

The main contribution of this work is to provide an enhanced framework for modeling special features of argumentation varying over time, which are relevant in many real-world situations. This proposal has also the aim of advancing in the integration of time and valuation in the context of argumentation systems. We are interested in preserving the generality of the approach as much as possible, keeping it independent from any particular representation language, and considering the internal structure of the arguments involved. Putting these developments in the theoretical context of computational argumentation, we will also present some properties and equivalence results for contrasting the expressivity of $E-T A F$ and $E-T A F^{*}$ with classical abstract argumentation.

We can summarize the rest of the paper following the steps just described. Next, in Section 2, we will introduce the basic elements of abstract argumentation; then, in Section 3, we will show how time availability of arguments affects abstract argumentation, and in Section 4, the extension of E-TAF will show the addition of availability intervals to attacks. Later, in Section 5, a framework for structured argumentation will be presented, and in Section 6 we will add temporal elements to these type of frameworks. We will end this work reviewing the related literature and offering some conclusions.

\section{Abstract Argumentation}

Dung presented in $[24,25]$ the notion of Abstract Argumentation Frameworks $(A F)$ as a way of concentrating on important characteristics of a defeasible argumentation system from a bare bones, general point of view. In these frameworks, an argument is considered as an abstract entity with unspecified internal structure, and whose role in the model is determined only by how it is related to other arguments through a relation of attack. This abstraction allows the definition of a number of general argumentation semantics based on acceptability, which then can be applied to any concrete argumentation system instantiating the $A F$. 
Definition 1 (Argumentation Framework [25]). An argumentation framework $(A F)$ is a pair $\langle A R, A t t s\rangle$, where $A R$ is a set of arguments, and Atts is a binary relation defined over $A R$ (representing attack), that is, Atts $\subseteq A R \times A R$.

Given an $A F$, an argument $A$ is considered acceptable if it can be defended from all the arguments that attack it (attackers) with arguments in $A R$. These intuitions are formalized in the following definitions, originally presented in [25].

Definition 2 (Acceptability). Let $A F=\langle A R, A t t s\rangle$ be an argumentation framework, then:

- $A$ set $S \subseteq A R$ is said to be conflict-free in $A F$ if there are no arguments $A, B \in S$ such that $(A, B) \in$ Atts.

- An argument $A \in A R$ is considered acceptable with respect to a set $S \subseteq$ $A R$ in $A F$ iff for each $B \in A R$ that attacks $A$ there exists an argument $C \in S$ such that $(C, B) \in$ Atts; it is also said that $B$ is attacked by $S$.

- A conflict-free set $S \subseteq A R$ is said to be admissible in AF iff each argument in $S$ is acceptable with respect to $S$.

- An admissible set $S \subseteq A R$ is a complete extension of AF iff $S$ contains each argument that is acceptable with respect to $S$.

From the above definitions, different semantics refining admissibility have been introduced by Dung [25]. Given $A F=\langle A R$, Atts $\rangle$ the following semantics were defined:

- preferred semantics: A set $E \subseteq A R$ is a preferred extension of $A F$ iff $E$ is a $\subseteq$-maximal admissible set; or equivalently is a $\subseteq$-maximal complete set. An argument $A \in A R$ is acceptable with respect to the preferred semantics iff $A$ is in every preferred extension $E$. It can be shown that there exists always at least one preferred extension.

- stable semantics: A set $E \subseteq A R$ is a stable extension of $A F$ iff $E$ is a conflict free set and $E$ attacks every argument in $A R \backslash E$. An argument $A \in A R$ is acceptable with respect to the stable semantics iff $A$ is in every stable extension $E$. Every stable extension is a preferred extension, but the converse does not hold.

- grounded semantics: A set $E \subseteq A R$ is the grounded extension of $A F$ iff $E$ is a $\subseteq$-minimal complete set. An argument $A \in A R$ is acceptable with respect to the grounded semantics iff $A$ is a member of the grounded extension $E$. There is always exactly one grounded extension, and this extension is a subset of all preferred and stable extensions.

Notice that regarding the arguments to be accepted by each semantics, we are following a skeptical approach, i.e., when more than one extension is possible we accept the arguments that belong to all of them. Dung [25] also presented a fixed-point characterization of the grounded semantics based on the characteristic function $F$ defined below. 
Definition 3. Let $\langle A R, A t t s\rangle$ be an AF. The associated characteristic function is defined as follows: $F: 2^{A R} \rightarrow 2^{A R}$ :

$$
F(S)=\operatorname{def}\{A \in A R \mid A \text { is acceptable w.r.t. } S\}
$$

The following proposition suggests how to compute the grounded extension associated with a finitary AF (i.e., such that each argument is attacked by at most a finite number of arguments) by iteratively applying the characteristic function starting from $\emptyset$.

Proposition 1 ([25]). Let $\langle A R$, Atts $\rangle$ be a finitary AF. Let $i \in \mathbb{N} \cup\{0\}$ such that $F^{i}(\emptyset)=F^{i+1}(\emptyset)$. Then $F^{i}(\emptyset)$ is the least fixed point of $F$, and corresponds to the grounded extension associated with the AF.

Example 1. Consider the $A F=\langle A R$, Atts $\rangle$ graphically represented in Fig. 1, where $A R=\{A, B, C, D, E, F, G, H, I, J, K\}$ and Atts $=\{(A, B),(B, C),(E, F),(F, D),(D, E)$, $(H, I),(I, H),(H, J),(I, J),(J, K)\}$.

The sets $E_{1}=\{A, C, G, H, K\}$ and $E_{2}=\{A, C, G, I, K\}$ are admissible and complete.
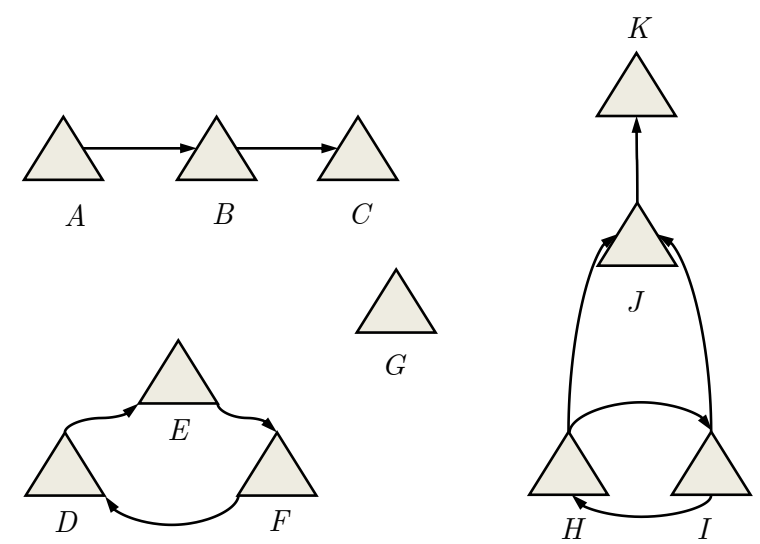

Figure 1: Argumentation Framework

Finally, $E_{1}$ and $E_{2}$ are the maximal sets verifying the previous conditions, and therefore they are preferred extensions of AR. As we can see, for a given argumentation framework there may exist multiple preferred extensions. The intersection of these sets, $\{A, C, G, K\}$, corresponds to the set of accepted arguments according to the preferred semantics.

In this example there is no stable extension, as there are cycles of odd length. When there are no cycles of odd length, the stable extensions coincide with the preferred extensions.

The set $E_{0}=\{A, C, G\}$ is admissible, since it defends all the arguments it contains. $E_{0}$ is also complete since it contains all the arguments in $A R$ defended by $E_{0}$. Finally, it can be verified that $E_{0}$ is the minimal set satisfying the previous conditions, and therefore it corresponds to the grounded extension of $A R$. 
Finally, the grounded extension for the example in Fig. 1 can be obtained by applying the fixed point characterization from Prop. 1.

$$
\begin{aligned}
& F^{0}(\emptyset)=\emptyset \\
& F^{1}(\emptyset)=F(\emptyset)=\{A, G\} \\
& F^{2}(\emptyset)=F(\{A, G\})=\{A, G, C\} \\
& F^{3}(\emptyset)=F(\{A, G, C\})=F^{2}(\emptyset)
\end{aligned}
$$

\section{Modeling Temporal Argumentation with TAF}

Modeling time has been a concern of researchers in Artificial Intelligence for many decades (see for instance [1] for a landmark paper in the area). Reviewing that research is out of the scope of this paper and we refer the interested reader to $[45,28]$. In the area of Argumentation in Artificial Intelligence, the first attempts to introduce temporal temporal argumentative reasoning can be traced to $[6,7,33,22]$. Next, we will recall the basis of [23].

Timed Abstract Frameworks (TAF) [22, 23] incorporate time to abstract frameworks adding that dimension to arguments; thus, arguments are valid only during specific intervals of time that are called availability intervals. Attacks between arguments are considered only when both the attacker and the attacked arguments are simultaneously available. Therefore, when identifying the set of acceptable arguments, the outcome associated with a given $T A F$ may vary accordingly to time.

To represent time, we assume that a correspondence was defined between the time line and the set of positive real numbers including 0 , represented as $\mathbb{R}^{+}=\{x \in \mathbb{R} \mid 0 \leq x\}$. A time interval, representing a period of time without interruptions, will be then represented as defined below. Notice that we use 'instead of ',' as a separator for legibility reasons.

Definition 4 (Time Interval). Given $a, b \in \mathbb{R}^{+}$, a time interval, or just an interval, is a set of positive real numbers. We consider four possible time intervals:

$$
\begin{aligned}
& (a-b)={ }_{\text {def }}\left\{x \in \mathbb{R}^{+} \mid a<x<b\right\} \\
& (a-b]={ }_{\text {def }}\left\{x \in \mathbb{R}^{+} \mid a<x \leq b\right\} \\
& {[a-b)={ }_{\text {def }}\left\{x \in \mathbb{R}^{+} \mid a \leq x<b\right\}} \\
& {[a-b]={ }_{\text {def }}\left\{x \in \mathbb{R}^{+} \mid a \leq x \leq b\right\}}
\end{aligned}
$$

As is usual, any of the intervals shown is considered empty if $b<a$, and the interval $[a-a]$ represents the point in time $\{a\}$. For the infinite endpoint, we use the symbol $+\infty$, as in $[a-+\infty)$, to indicate that there is no upper bound for the interval, and an interval containing this symbol will always be closed by ")".

To model discontinuous periods of time we introduce the notion of time intervals set. Although a time intervals set suggests a representation as a set of sets (set of intervals), we chose a flattened representation as a set of reals (the set of all 
real numbers contained in any of the individual time intervals). Thusly, we can directly apply traditional set operations and relations on time intervals sets.

Definition 5 (Time Intervals Set). A time intervals set, or just intervals set, is a finite set $S$ of time intervals.

Note that $S \subseteq \mathbb{R}^{+}$; when convenient, we will use the set of sets notation for time intervals sets. Concretely, a time interval set $S$ will be denoted as the set of all disjoint and $\subseteq$-maximal individual intervals included in the set. For instance, we will use $\{(1-3],[4.5-8)\}$ to denote the time interval set $(1-3] \cup[4.5-8)$. We now formally introduce the notion of Timed Argumentation Framework, which extends Dung's $A F$ by incorporating the availability function. This function will be used to capture those time intervals where arguments are available.

Definition 6 (Timed Argumentation Framework). A timed argumentation framework (or simply TAF) is a triple $\Phi=\langle A R, A t t s, A v\rangle$, where $A R$ is a set of arguments, Atts is a binary relation defined over AR (representing attack), and $A v: A R \longrightarrow \wp\left(\mathbb{R}^{+}\right)$is an availability function for timed arguments, such that $A v(A)$ is the set of availability intervals of an argument $A$.

Notice that we assume that a set $S$ of intervals is equivalent to the set of time points associated with every interval in $S$. Thus, the image for $A v$ is $\wp\left(\mathbb{R}^{+}\right)$.

Example 2. Figure 2 depicts the TAF $\Phi=\langle A R$, Atts, Av $\rangle$ where:

$$
\begin{aligned}
& A R=\{A, B, C, D, E, F, G, H, I, J, K\} \\
& A t t s=\{(A, B),(B, C),(E, F),(F, D),(D, E),(H, I),(I, H),(H, J,),(I, J,),(J, K)\} . \\
& A v=\{(A,\{[0-30]\}) ;(B,\{[10-50]\}) ;(C,\{[0-60]\}) ;(D,\{[10-30]\}) ;(E,\{[10-30]\}) ; \\
&(F,\{[0-30]\}) ;(G,\{[0-90]\}) ;(H,\{[10-50]\}) ;(I,\{[20-30]\}) ;(J,\{[20-30]\}) ; \\
&(K,\{[20-30]\})\}
\end{aligned}
$$

The following definitions formalize argument acceptability in $T A F$, and are extensions of the acceptability notions presented in section 2 for $A F$. Firstly, we present the notion of $t$-profile, binding an argument to a set of time intervals, which constitutes a fundamental component for time-based acceptability.

Definition 7 (T-Profile). Let $\Phi=\langle A R, A t t s, A v\rangle$ be a TAF. A timed argument profile for $A$ in $\Phi$, or just t-profile for $A$, is a pair $\left(A, \mathcal{T}_{A}\right)$ where $A \in A R$ and and $\mathcal{T}_{A}$ is a set of time intervals where $A$ is available, i.e., $\mathcal{T}_{A} \subseteq A v(A)$. The t-profile $(A, A v(A))$ is called the basic t-profile of $A$.

Definition 8 (Collection of T-Profiles). Let $\Phi=\langle A R$, Atts, Av $\rangle$ be a TAF . Let $\left(X_{1}, \mathcal{T}_{X_{1}}\right),\left(X_{2}, \mathcal{T}_{X_{2}}\right), \ldots,\left(X_{n}, \mathcal{T}_{X_{n}}\right)$ be t-profiles. The set $S=\left\{\left(X_{1}, \mathcal{T}_{X_{1}}\right)\right.$, $\left.\left(X_{2}, \mathcal{T}_{X_{2}}\right), \ldots,\left(X_{n}, \mathcal{T}_{X_{n}}\right)\right\}$ is a collection of t-profiles iff it verifies the following conditions:

i) $X_{i} \neq X_{j}$ for all $i, j$ such that $i \neq j, 1 \leq i, j \leq n$.

ii) $\mathcal{T}_{X_{i}} \neq \emptyset$, for all $i$ such that $1 \leq i \leq n$. 


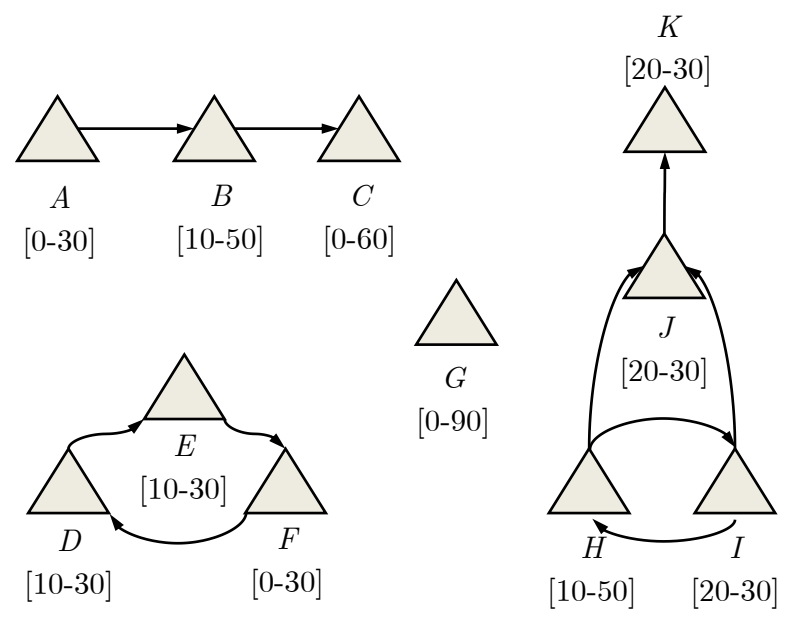

Figure 2: $T A F$ corresponding to example 2

Since the availability of arguments varies in time, the acceptability of a given argument $A$ will also vary in time. To do this, it is necessary to introduce two new concepts corresponding to the intersection and inclusion of t-profiles, denoted as t-intersections and t-inclusions, formalized below:

Definition 9 (t-intersection). Let $\Phi=\langle A R, A t t s, A v\rangle$ be a TAF. Let $S_{1}$ and $S_{2}$ be two collections of t-profiles. We define the t-intersection of $S_{1}$ and $S_{2}$, denoted $S_{1} \cap_{t} S_{2}$, as the collection of t-profiles such that:

$$
S_{1} \cap_{t} S_{2}=\left\{\left(X, \mathcal{T}_{X} \cap \mathcal{T}_{X^{\prime}}\right) \mid\left(X, \mathcal{T}_{X}\right) \in S_{1},\left(X, \mathcal{T}_{X^{\prime}}\right) \in S_{2}, \text { and } \mathcal{T}_{X} \cap \mathcal{T}_{X^{\prime}} \neq \emptyset\right\}
$$

Definition 10 (t-inclusion). Let $S_{1}$ and $S_{2}$ be two collections of t-profiles. We say that $S_{1}$ is t-included in $S_{2}$, denoted as $S_{1} \subseteq_{t} S_{2}$, if for any t-profile $\left(X, \mathcal{T}_{X}\right) \in S_{1}$ there exists a $t$-profile $\left(X, \mathcal{T}_{X}^{\prime}\right) \in S_{2}$ such that $\mathcal{T}_{X} \subseteq \mathcal{T}_{X}^{\prime}$.

The following definitions reformulate Dung's original formalization for abstract argumentation considering $t$-profiles instead of arguments. First, we will extend the notion of conflict-free set to $t$-profiles in a $T A F$, and then we will define how an argument is defended over time.

Definition 11 ( $t$-conflict-free). A collection $S$ of t-profiles is said to be $t$-conflict-free in a TAF $\Phi$ if there are no t-profiles $\left(A, \mathcal{T}_{A}\right),\left(B, \mathcal{T}_{B}\right) \in S$ such that $(A, B) \in$ Atts and $\mathcal{T}_{A} \cap \mathcal{T}_{B} \neq \emptyset$.

Definition 12 (Defense of $A$ from $B$ by $S$ ). Given $\Phi=\langle A R$, Atts, Av $\rangle$, let $S$ be a $t$-conflict-free collection of $t$-profiles, and $A, B \in A R$, with $t$-profiles $(A, A v(A))$ and $(B, A v(B))$ respectively. The defense $t$-profile of $A$ from $B$ w.r.t. $S$, denoted as $\mathcal{T}_{(A \mid S)}^{B}$ is defined as follows:

$$
\mathcal{T}_{(A \mid S)}^{B}={ }_{\text {def }} A v(A) \cap \bigcup_{C \in\left\{X \mid\left(X, \mathcal{T}_{X}\right) \in S,(X, B) \in A t t s\right\}} A v(B) \cap \mathcal{T}_{C}
$$


Intuitively, $A$ is defended from the attack of $B$ when $B$ is not available, plus those intervals where the attacker $B$ is available but it is in turn attacked by an argument $C$ in the set $S$. The following definition captures the defense profile of $A$, but considering all its attacking arguments.

Definition 13 (Acceptable t-profile of $A$ w.r.t. $S$ ). Let $\Phi=\langle A R, A t t s, A v\rangle$ be a TAF, let $S$ be a collection of $t$-profiles. The acceptable t-profile for $A$ w.r.t. $S$, denoted as $\mathcal{T}_{(A \mid S)}$ is defined as follows:

$$
\mathcal{T}_{(A \mid S)}={ }_{\operatorname{def}} \bigcap_{B \in\{X \mid(X, A) \in A t t s\}}(A v(A) \backslash A v(B)) \cup \mathcal{T}_{(A \mid S)}^{B}
$$

Where $\mathcal{T}_{(A \mid S)}^{B}$ is the time interval where $A$ is defended of its attacker $B$ by $S$. Then, the intersection of all time intervals in which $A$ is defended from each of its attackers by the set $S$, is the time interval where $A$ is available and is acceptable with respect to $S$.

Now, we introduce versions of admissibility and completeness suitably extended for the context of temporal argumentation frameworks.

Definition 14 ( $t$-admissible/ $t$-complete). Given the TAF $\Phi=\langle A R, A t t s, A v\rangle$ :

- A collection $S$ of $t$-profiles is $t$-admissible in $\Phi$ iff for all $\left(A, \mathcal{T}_{(A \mid S)}\right) \in S$ it holds $\left(A, \mathcal{T}_{(A \mid S)}\right)$ is an acceptable t-profile of $A$ w.r.t. $S$.

- A t-admissible collection $S$ is a t-complete extension of $\Phi$ iff $S$ contains all the $t$-profiles that are acceptable with respect to $S$.

As in the framework proposed by Dung, we can define now the acceptability semantics for $T A F$.

Definition 15. Let $\Phi=\langle A R, A t t s, A v\rangle$ be a TAF, we can define the t-preferred semantics, $t$-stable semantics, and t-grounded semantics as follows:

- $t$-preferred semantics: A collection $E$ of $t$-profiles is a $t$-preferred extension of $\Phi$ iff $E$ is $a \subseteq_{t}$-maximal $t$-admissible collection of $t$-profiles.

- $t$-stable semantics: Given a collection $E=\left\{\left(Y_{i}, \mathcal{T}_{\left(Y_{i} \mid E\right)}\right) \mid 1 \leq i \leq n\right\}$ of $t$-profiles that satisfies $t$-conflict-freeness, $E$ is a t-stable extension of $\Phi$ iff for all $X \in A R \backslash\left\{Y_{i} \mid 1 \leq i \leq n\right\}$ with $t$-profile $\left(X, \mathcal{T}_{X}\right)$ it holds that:

$$
\mathcal{T}_{X} \backslash \bigcup_{i=1}^{n} \mathcal{T}_{\left(Y_{i} \mid E\right)}=\emptyset
$$

- $t$-grounded semantics: A collection $E$ of t-profiles is the t-grounded extension of $\Phi$ iff $E$ is a $\subseteq_{t}$-minimal t-complete collection of $t$-profiles.

Regarding the arguments to be sanctioned in each semantics, we will take the skeptical approach, i.e., when more than one extension exist we will accept the arguments that belong to all of them. Skeptical acceptability for the three semantics described can be defined as follows: 
Definition 16. Let $\Phi=\langle A R, A t t s, A v\rangle$ be a TAF, and let $\left\{E_{1}, E_{2}, \ldots, E_{n}\right\}$ be the set of $t$-preferred ( $t$-stable) extensions of $\Phi$. An argument $A \in A R$ is acceptable under $t$-preferred ( $t$-stable) semantics with a $t$-profile $\left(A, \mathcal{T}_{A}\right)$ iff $\mathcal{T}_{A}=\bigcap_{i=1}^{n} \mathcal{T}_{\left(A \mid E_{i}\right)}$ and $\mathcal{T}_{A} \neq \emptyset$.

An argument $A \in A R$ is acceptable under t-grounded semantics in the time interval $\mathcal{T}_{(A \mid E)}$ iff $\left(A, \mathcal{T}_{(A \mid E)}\right) \in E$, where $E$ is the t-grounded extension.

As in abstract frameworks, the equivalence between a $\subseteq_{t}$-maximal t-complete extension and $\subseteq_{t}$-maximal t-admissible sets holds; and, t-preferred extensions are equivalently defined as t-complete $\subseteq_{t}$-maximal sets. The formal statement of these equivalences appears below; the proof follows directly from the definitions.

Proposition 2. Let $\Phi=\langle A R, A t t s, A v\rangle$ be a TAF, and let $E$ be a collection of t-profiles. Then, $E$ is a $\subseteq_{t}$-maximal $t$-admissible collection of t-profiles iff $E$ is $a \subseteq_{t}$-maximal t-complete extension.

Also, the $t$-stable, $t$-preferred, and $t$-complete semantics admit multiple extensions, whereas the $t$-grounded semantics ascribes a single extension to a given argument system. In particular, the fixed point characterization for grounded semantics proposed by Dung can be directly applied to TAF by considering the following modified version of the characteristic function.

Definition 17. Let $\langle A R, A t t s, A v\rangle$ be a TAF. Let $S$ be a collection of $t$-profiles. The associated characteristic function is defined as follows:

$$
F(S)=_{\text {def }}\left\{\left(A, \mathcal{T}_{(A \mid S)}\right) \mid A \in A R \text { and }\left(A, \mathcal{T}_{(A \mid S)}\right) \text { is the acceptable t-profile of } A \text { w.r.t. } S\right\}
$$

Note that there exiat always an $t$-grounded extension, and the $t$-grounded extension is a t-subset of all $t$-preferred and $t$-stable extensions; also, a $t$-stable extension is also a $t$-preferred extension and a $t$-preferred extension is also a t-complete extension.

Example 3. Suppose we want to establish the acceptability of $C$ in the TAF $\Phi$ presented in example 2. As it was shown in example 1, by considering only the Atts relation we could conclude that the argument $C$ is acceptable.

Let us analyze the acceptability of the argument $C$ in the time interval $A v(C)$ in each semantics of TAF. First we analyze the t-preferred extension, next the $t$-stable extension and finally the t-grounded extension. The collections of t-profiles

$$
\begin{aligned}
S_{1}=\{(A,\{[0-30]\}) ;(B,\{(30-50]\}) ;(C,\{[0-30],(50-60]\}) ;(F,\{[0-10)\}) ; \\
(G,\{[0-90]\}) ;(H,\{[10-50]\}) ;(K,\{[20-30]\})\} . \\
S_{2}=\{(A,\{[0-30]\}) ;(B,\{(30-50]\}) ;(C,\{[0-30],(50-60]\}) ;(F,\{[0-10)\}) ; \\
(G,\{[0-90]\}) ;(H,\{[10-20),(30-50]\}) ;(I,\{[20-30]\}) ;(K,\{[20-30]\})\} .
\end{aligned}
$$

are $t$-admissible, since they defend every t-profile they contain, i.e., for the specific time intervals involved. $S_{1}$ and $S_{2}$ are also t-complete since they include all the $t$ profiles defended by $C$ in their associated time intervals. Finally, it can be verified that $S_{1}$ and $S_{2}$ are the maximal sets satisfying the previous conditions, and therefore both of them correspond to the t-preferred extension; also, as we can see, it is possible to obtain multiple t-preferred extensions. The intersection of these collections results in a collection of accepted t-profiles. For the particular example given above, we have 


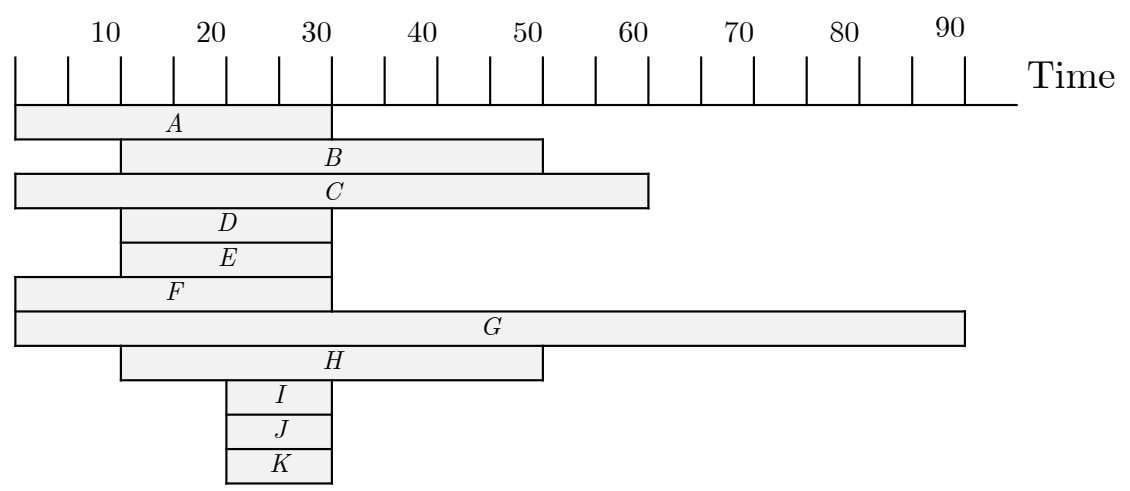

Figure 3: Representation of the arguments associated with Ex. 3 in a time line

$$
\begin{aligned}
S_{1} \cap_{t} S_{2}=\{(A,\{[0-30]\}) ;(B,\{(30-50]\}) ;(C,\{[0-30],(50-60]\}) ;(F,\{[0-10)\}) ; \\
\\
(G,\{[0-90]\}) ;(H,\{[10-20),(30-50]\}) ;(K,\{[20-30]\})\} .
\end{aligned}
$$

In this example there is no t-stable extension, as there are odd length cycles. When there are no cycles of odd length, the t-stable extension coincides with the t-preferred extension. For example, suppose now that we want to establish the acceptability of $C$ in the TAF $\Phi$ presented in example 2. As shown in example 1, by considering only the Atts relation we could conclude that the argument $C$ is acceptable. Let us obtain the $t$-grounded extension of $\Phi$ by applying the fixed point characterization.

$$
\begin{aligned}
F^{0}(\emptyset)=\emptyset & \emptyset \\
F^{1}(\emptyset)= & \begin{aligned}
(A,\{[0-30]\}) ;(B,\{(30-50]\}) ;(C,\{[0-10),(50-60]\}) ; \\
\quad(G,\{[0-90]\}) ;(H,\{[10-20),(30-50]\}) ;(F,\{[0-10)\})\}
\end{aligned} \\
F^{2}(\emptyset)= & \begin{aligned}
(A,\{[0-30]\}) ;(B,\{(30-50]\}) ;(C,\{[0-30],(50-60]\}) ; \\
\quad(G,\{[0-90]\}) ;(H,\{[10-20),(30-50]\}) ;(F,\{[0-10)\})\}
\end{aligned} \\
F^{3}(\emptyset)= & F^{2}(\emptyset)
\end{aligned}
$$

Consequently, $F^{2}(\emptyset)$ is the $t$-grounded extension of $\Phi$. Next, we describe how the temporal availability of $C$ was obtained in $F^{2}(\emptyset)$ by applying the definitions 12 and 13 starting from $F^{1}(\emptyset)$. By applying definition 12 :

$$
\begin{aligned}
\mathcal{T}_{\left(C \mid F^{1}(\emptyset)\right)}^{B} & =A v(C) \cap \bigcup_{\left(A, \mathcal{T}_{A}\right) \in\left\{\left(X, \mathcal{T}_{X}\right) \mid\left(X, \mathcal{T}_{X}\right) \in F^{1}(\emptyset),(X, B) \in A t t s\right\}}\left(A v(B) \cap \mathcal{T}_{A}\right)= \\
& =\{[0-60]\} \cap(\{[10-50]\} \cap\{[0-30]\})= \\
& =\{[0-60)\} \cap[10-30]= \\
& =\{[10-30]\}
\end{aligned}
$$

By definition 13 :

$$
\mathcal{T}_{\left(C \mid F^{1}(\emptyset)\right)}=(A v(C) \backslash A v(B)) \cup \mathcal{T}_{\left(C \mid F^{1}(\emptyset)\right)}^{B}=
$$




$$
=\{[0-10),(50-60]\} \cup\{[10-30]\}=\{[0-30],(50-60]\}
$$

\section{E-TAF: Extending TAF with time intervals for attacks}

Our presentation up to this point assigns a temporal availability to arguments, indicating the temporal interval where the argument is available to support its conclusion; but, the effectiveness of the argument is still static, i.e., its ability to support its conclusion does not change in the time interval in which is available. However, there are domains where the effectiveness of the argument can vary in time; for example, the reliability of the information used for recommending a particular investment changes as time passes.

Considering this fluctuation of the effectiveness through the time, it is clear that the success of an attack between two arguments depends on the time interval in which that attack occurs; therefore, the success of an attack will depend on the time subinterval when the attacker is more effective than the argument receiving that attack. This situation is local to the pair of arguments in conflict; simultaneously, in its time interval, the attacker could be performing an attack to a different argument but its attack being effective in a different subinterval of its availability. Therefore, in the case described, two attacks performed by the same argument have different time intervals of effectiveness associated; for this reason, it is necessary to associate time to the argument and the attack separately.

Associating time intervals to attacks is an important extension of the representation capabilities of a TAF. This separation allows to model what-if scenarios to analyze different situations where, for instance, the effectiveness of attacks and the availability of arguments and attacks change. We will illustrate these situations below.

We now introduce Extended Temporal Argumentation Frameworks (E-TAF) adding that capability to TAFs; thus, this framework takes into account not only the availability of the arguments as in TAFs but adds the consideration of the availability of the attacks each argument delivers. That is, as the example that follows illustrates, an argument might be available for consideration but the attack it carries might not.

In certain situations regulated by law, there is the concept of statute of limitations. This is a legislative act that sets a time limit on legal action in certain cases; that is, an enactment in a common law legal system that sets the maximum time after an event that legal proceedings based on that event may be initiated. All systems of law have statutes restricting the time within which legal proceedings may be brought. The periods prescribed may vary according to how serious the offense was, some crimes never prescribe, and the time limitations can be extended in special situations. ${ }^{1}$ One reason for

\footnotetext{
${ }^{1}$ The statute of limitations may vary in different countries; for the case of the U.S. see for instance http://www.statuteoflimitations.net
} 
having a statute of limitations is that over time evidence can be corrupted or disappear; thus, the best time to bring a lawsuit is while the evidence is not lost and as close as possible to the alleged illegal behavior. Another reason is that people want to get on with their lives and not have legal battles from their past come up unexpectedly. The injured party has a responsibility to quickly bring about charges so that the process can begin. Examples of offenses regulated by statute of limitation laws are fraud, medical malpractice, debt collection, wrongful death, etc.

Consider the following situation: John has left debts unpaid in Alabama, US, during 2008. He has canceled them in 2009, but he paid his debts with counterfeited US dollars, committing fraud. This fraud was detected on Jan 1 , 2010. A possible argument exchange for prosecuting John could be as follows:

- Arg $_{1}:$ (Plaintiff) John left debts unpaid in Alabama in 2008, therefore the

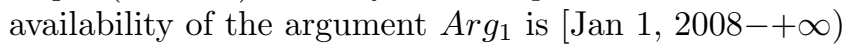

- Arg $_{2}$ : (Defendant) John paid all his debts in Alabama for 2008, thus Arg $_{2}$

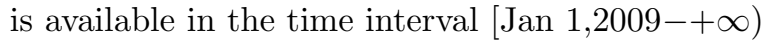

- Arg $_{3}:$ (Plaintiff) John did not cancel his debts in Alabama for 2008, as he paid them with counterfeited US dollars, committing fraud, so $\mathrm{Arg}_{3}$ is

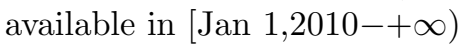

According to Alabama's statute of limitations, the attack from $\mathrm{Arg}_{3}$ to $\mathrm{Arg}_{2}$ would be valid just until Jan 1, 2012 (that is, 2 years from the moment it was discovered). Note that $\mathrm{Arg}_{3}$ is valid by itself (as the fraud was committed anyway), but the statute of limitations imposes a time-out on the attack relationship between arguments $\mathrm{Arg}_{3}$ and $\mathrm{Arg}_{2}$; thus, John would be not liable of prosecution for committing fraud if the dialogue would have taken place in 2012, as the attack from $\mathrm{Arg}_{3}$ to $\mathrm{Arg}_{2}$ would cease to be applicable.

Next we formalize the definition of the proposed extension of $T A F$, which provides the elements required to capture timed attacks between timed arguments.

Definition 18 (Extended TAF). An extended timed abstract argumentation framework (or simply E-TAF) is a 4-tuple $\Theta=\langle A R$, Atts, ArgAv, AttAv $\rangle$ where:

i) $A R$ is a set of arguments,

ii) Atts is a binary relation defined over AR representing attack,

iii) $\operatorname{Arg} A v: A R \longrightarrow \wp\left(\mathbb{R}^{+}\right)$is the availability function for timed arguments, and

iv) AttAv : Atts $\longrightarrow \wp\left(\mathbb{R}^{+}\right)$is the availability function for timed attacks, where $\operatorname{AttAv}((A, B)) \subseteq \operatorname{Arg} A v(A) \cap \operatorname{Arg} A v(B)$. 
Items $(i),(i i)$, and (iii) represent the TAF part of the extended framework. The item mentioned in $i v), \operatorname{Att} A v((A, B)) \subseteq \operatorname{Arg} A v(A) \cap \operatorname{Arg} A v(B)$, ensures that the availability of the attack cannot exceed the availability of the arguments involved, and $\mathbb{R}^{+}$is the set of positive real numbers plus 0 . To simplify the notation, we will continue using $A v(A)$ for $\operatorname{Arg} A v(A)$ and we will use $\mathcal{T}_{(A, B)}$ for $\operatorname{Att} A v((A, B))$.

Example 4. Consider the E-TAF $\Theta=\langle A R$, Atts, ArgAv, AttAv $\rangle$ in Fig. 4

$$
\begin{aligned}
& A R=\{A, B, C, D, E, F, G, H, I, J, K\} \\
& \text { Atts }=\{(A, B),(B, C),(E, F),(F, D),(D, E),(H, I),(I, H)\} . \\
& A r g A v=\{(A,\{[0-30]\}) ;(B,\{[10-50]\}) ;(C,\{[0-60]\}) ;(D,\{[10-30]\}) ; \\
&(E,\{[10-30]\}) ;(F,\{[0-30]\}) ;(G,\{[0-90]\}) ;(H,\{[10-50]\}) ; \\
&(I,\{[20-30]\}) ;(J,\{[20-30]\}) ;(K,\{[20-30]\})\} \\
& A t t A v=\{((A, B),\{[15-30]\}) ;((B, C),\{20-50]\}) ;((H, I),\{[20-25]\}) ; \\
&((I, H),\{[20-30]\}) ;((D, E),\{[20-30]\}) ;((E, F),\{[20-30]\}) ; \\
&((F, D),\{[20-30]\}) ;((H, J),\{[20-25]\}) ;((I, J),\{[20-25]\}) ; \\
&((J, K),\{[20-25]\})\}
\end{aligned}
$$
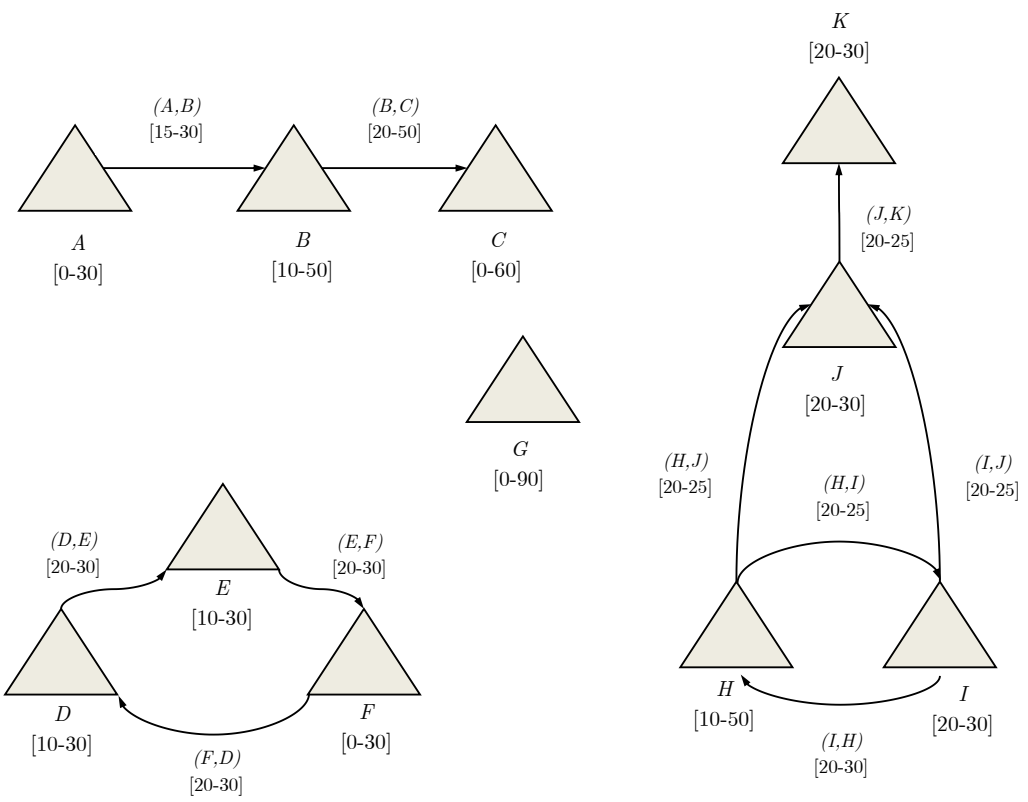

Figure 4: An example of E-TAF

The following definitions are extensions of the definitions 11, 12 and 13, taking into account the availability of attacks. 
Definition 19 (at-conflict-freeness). Let $\Theta=\langle A R, A t t s, A r g A v, A t t A v\rangle$ be an $E$-TAF. A set $S$ of t-profiles is called at-conflict-free in $\Theta$ if there are no t-profiles $\left(A, \mathcal{T}_{A}\right),\left(B, \mathcal{T}_{B}\right) \in S$ such that $(A, B) \in$ Atts and

$$
\mathcal{T}_{A} \cap \mathcal{T}_{B} \cap \mathcal{T}_{(A, B)} \neq \emptyset
$$

Definition 20 (Defense of $A$ from $B$ by $S$ ). Given an $E$-TAF defined as $\Theta=\langle A R, A t t s, A r g A v, A t t A v\rangle, S$ a collection of t-profiles, and $A, B \in A R$, with $t$-profiles $(A, A v(A))$ and $(B, A v(B))$ respectively. The defense $t$-profile of $A$ from $B$ w.r.t. $S$, denoted as $\mathcal{T}_{(A \mid S)}^{B}$ is defined as follows:

$$
\mathcal{T}_{(A \mid S)}^{B}={ }_{\text {def }} \bigcup_{\left(C, \mathcal{T}_{C}\right) \in\left\{\left(X, \mathcal{T}_{X}\right) \mid\left(X, \mathcal{T}_{X}\right) \in S,(X, B) \in A t t s\right\}}\left(\mathcal{T}_{(B, A)} \cap \mathcal{T}_{(C, B)} \cap \mathcal{T}_{C}\right)
$$

From the previous definitions, the notion of acceptable t-profile of $A$ w.r.t. $S$ in $E-T A F$ coincides with the corresponding definition in $T A F$.

Definition 21 (Acceptable t-profile of $A$ w.r.t. $S$ ). Given an E-TAF $\Theta=$ $\langle A R, A t t s, A r g A v, A t t A v\rangle$, let $S$ be a set of t-profiles. The acceptable t-profile for $A$ w.r.t. to $S$, denoted as $\mathcal{T}_{(A \mid S)}$ is defined as follows:

$$
\mathcal{T}_{(A \mid S)}={ }_{\text {def }} \bigcap_{B \in\{X \mid(X, A) \in A t t s\}}\left(A v(A) \backslash \mathcal{T}_{(B, A)}\right) \cup \mathcal{T}_{(A \mid S)}^{B}
$$

Thus, this definition reflects that the intersection of all time intervals in which $A$ is defended from each of its attackers by the set $\mathrm{S}$, is the time interval where $A$ is available and is acceptable with respect to $S$. The acceptability formalization for $T A F$ directly applies to $E-T A F$. In the same way that the original definition of $t$-conflict-free was recast into at-conflict-free, we proceed to characterize the notions of at-admissible and at-complete. Given the $\Theta=\langle A R, A t t s, A r g A v, A t t A v\rangle$, we can define now the semantics for $E-T A F$ as follows:

- at-preferred semantics: A collection $E$ of $t$-profiles is an at-preferred extension of $\Theta$ iff $E$ is a $\subseteq_{t}$-maximal at-admissible collection of t-profiles.

- at-stable semantics: Given a collection $E=\left\{\left(Y_{i}, \mathcal{T}_{\left(Y_{i} \mid E\right)}\right) \mid 1 \leq i \leq n\right\}$ of $t$-profiles that satisfies at-conflict-freeness, $E$ is a at-stable extension of $\Theta$ iff for all $X \in A R \backslash\left\{Y_{i} \mid 1 \leq i \leq n\right\}$ with $t$-profile $\left(X, \mathcal{T}_{X}\right)$ it holds that $\mathcal{T}_{X} \backslash \bigcup_{i=1}^{n} \mathcal{T}_{\left(Y_{i} \mid E\right)}=\emptyset$.

- at-grounded semantics: A collection $E$ of t-profiles is the at-grounded extension of $\Theta$ iff $E$ is a $\subseteq_{t}$-minimal at-complete collection of t-profiles.

Regarding the arguments to be accepted in each semantics, we will take the skeptical approach, i.e., when more than one extension is possible we will accept the arguments that belong to all of them in the common time interval they are active. Skeptical acceptability for the three semantics described can be defined as follows: 
Definition 22. Let $\Theta=\langle A R, A t t s, A r g A v, A t t A v\rangle$ be an E-TAF, and let $\left\{E_{1}, E_{2}, \ldots, E_{n}\right\}$ be the set of at-preferred (at-stable) extensions of $\Theta$. An argument $A \in A R$ acceptable under at-preferred (at-stable) semantics with a t-profile $\left(A, \mathcal{T}_{A}\right)$ iff $\mathcal{T}_{A}=\bigcap_{i=1}^{n} \mathcal{T}_{\left(A \mid E_{i}\right)}$ and $\mathcal{T}_{A} \neq \emptyset$.

An argument $A \in A R$ is acceptable under at-grounded semantics in the time interval $\mathcal{T}_{(A \mid E)}$ iff $\left(A, \mathcal{T}_{(A \mid E)}\right) \in E$, where $E$ is the at-grounded extension.

Extending the notion presented in proposition 2, the equivalence between a $\subseteq_{t}$-maximal at-complete extension and $\subseteq_{t}$-maximal at-admissible collections of t-profiles holds; and, at-complete extensions are equivalently defined as atcomplete $\subseteq_{t}$-maximal collections of t-profiles. The formal statement of these equivalences appears below; the proof follows directly from the definitions.

Proposition 3. Let $\Theta=\langle A R, A t t s, A r g A v, A t t A v\rangle$ be an $E-T A F$, and let $E$ be a collection of t-profiles. Then, $E$ is a $\subseteq_{t}$-maximal at-admissible collection of t-profiles iff $E$ is $\subseteq_{t}$-maximal at-complete extension.

The fixed point characterization for grounded semantics proposed by Dung can be applied to $E-T A F$ just by considering the following modified version of the characteristic function.

Definition 23 (Characteristic function). The associated characteristic function for $\Theta=\langle A R$, Atts, ArgAv, AttAv $\rangle$ is defined as follows:

$F(S)=_{\text {def }}\left\{\left(A, \mathcal{T}_{(A \mid S)}\right) \mid A \in A R\right.$ and $\left(A, \mathcal{T}_{(A \mid S)}\right)$ is the acceptable $t$-profile of $A$ w.r.t. $\left.S\right\}$

The relations between at-grounded extensions, at-preferred extensions, at-stable extensions, and at-complete extensions is given in the following proposition.

Proposition 4. Let $\Theta=\langle A R, A t t s, A r g A v, A t t A v\rangle$ be an E-TAF, then:

(1) There always exists an at-grounded extension.

(2) An at-preferred extension is also an at-complete extension.

(3) An at-stable extension is also an at-preferred extension.

(4) The at-grounded extension is a t-subset of all at-preferred and at-stable extensions.

Example 5. Suppose we want to establish the acceptability of $C$ in the E-TAF $\Theta$ presented in the Figure 4. As shown in example 1, by considering only the relation Atts we could say that the argument $C$ is acceptable.

Now we analyze the acceptability of the argument $C$ in the time interval $A v(C)$ in each semantics of E-TAF. First we analyze the at-preferred extension, next the at-stable extension and finally the at-grounded extension. The sets of t-profiles are at-admissible, since they defend every t-profile that they contain, i.e., for the specific time intervals involved.

$$
\begin{aligned}
S_{1}=\{( & (A,\{[0-30]\}) ;(B,\{[10-15),(30-50]\}) ;(C,\{[0-30],(50-60]\}) ; \\
& (F,\{[0-20)\}) ;(D,\{[10-20)\}) ;(E,\{[10-20)\}) ;(G,[0-90]\}) ; \\
& (H,\{[10-25],(30-50]\}) ;(I\{(25-30]\}) ;(J,\{(25-30]\}) ;(K,\{[20-30]\})\}
\end{aligned}
$$



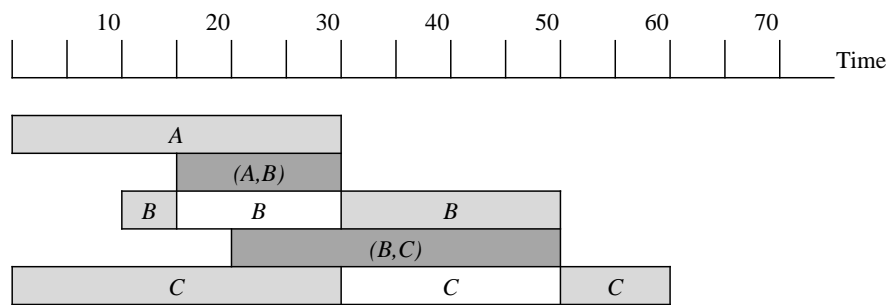

Figure 5: Representation of the temporal attack relations

$$
\begin{aligned}
S_{2}=\{ & (A,\{[0-30]\}) ;(B,\{[10-15),(30-50]\}) ;(C,\{[0-30],(50-60]\}) ; \\
& (F,\{[0-20)\}) ;(D,\{[10-20)\}) ;(E,\{[10-20)\}) ;(G,\{[0-90]\}) ; \\
& (H,\{[10-20),(30-50]\}) ;(I\{[20-30]\}) ;(J,\{(25-30]\}) ;(K,\{[20-30]\})\}
\end{aligned}
$$

$S_{1}$ and $S_{2}$ are also at-complete since they include all the t-profiles defended by $C$ in their associated time intervals. Finally, it can be verified that $S_{1}$ and $S_{2}$ are the maximal sets satisfying the previous conditions, and therefore both of them correspond to the at-preferred extension. As we can see, its possible to obtain multiple at-preferred extensions. The intersection of these sets results the set of accepted t-profiles, then

$$
\begin{aligned}
S_{1} \cap_{t} S_{2}=\{ & (A,\{[0-30]\}) ;(B,\{[10-15),(30-50]\}) ;(C,\{[0-30],(50-60]\}) ; \\
& (F,\{[0-20)\}) ;(D,\{[10-20)\}) ;(E,\{[10-20)\}) ;(G,\{[0-90]\}) ; \\
& (H,\{[10-20),(30-50]\}) ;(I\{(25-30]\}) ;(J,\{(25-30]\}) ;(K,\{[20-30]\})\}
\end{aligned}
$$

In this example there is no at-stable extension, as there are odd length cycles. If there is no cycle of odd length, the at-stable extension coincides with the at-preferred extension.

Suppose now we want to establish the acceptability of $C$ in the $E$-TAF $\Theta$ presented in example 4. As shown in the AF of Example 1, by considering only the Atts relation we could say that the argument $C$ is acceptable. Let us obtain the at-grounded extension of $\Theta$ by applying the fixed point characterization.

$$
\begin{aligned}
F^{0}(\emptyset)=\emptyset & \\
F^{1}(\emptyset)=\{ & (A,\{[0-30]\}) ;(B,\{[10-15)(30-50]\}) ;(C,\{[0-20),(50-60]\}) ; \\
& (G,\{[0-90]\}) ;(H,\{[10-20),(30-50]\}) ;(I,\{(25-30]\}) ; \\
& (J,\{(25-30]\}) ;(K,\{(25-30]\}) ;(F,\{[0-20)\}) ;(D,\{[10-20)\}) ; \\
& (E,\{[10-20)\})\} \\
F^{2}(\emptyset)=\{(A,\{[0-30]\}) ;(B,\{[10-15)(30-50]\}) ;(C,\{[0-30],(50-60]\}) ; & (G,\{[0-90]\}) ;(H,\{[10-20),(30-50]\}) ;(I,\{(25-30]\}) ; \\
& (J,\{(25-30]\}) ;(K,\{(25-30]\}) ;(F,\{[0-20)\}) ;(D,\{[10-20)\}) ; \\
& (E,\{[10-20)\})\} \\
F^{3}(\emptyset)= & F^{2}(\emptyset)
\end{aligned}
$$

Consequently, $F^{2}(\emptyset)$ is the at-grounded extension of $\Theta$.

Next, we describe how the temporal availability of $C$ was obtained in $F^{2}(\emptyset)$ by applying the definitions 20 and 21 from $F^{1}(\emptyset)$. 
By applying definition 20:

$$
\begin{aligned}
\mathcal{T}_{\left(C \mid F^{1}(\emptyset)\right)}^{B} & \bigcup \bigcup \\
& =\left\{\left[\mathcal{T}_{(B, C)} \cap \mathcal{T}_{(A, B)} \cap A v(A)\right)=\right. \\
= & \{[20-30]\}
\end{aligned}
$$

Applying definition 21 we obtain:

$\mathcal{T}_{\left(A \mid F^{1}(\emptyset)\right)}=\left(A v(C) \backslash \mathcal{T}_{(B, C)}\right) \cup \mathcal{T}_{\left(C \mid F^{1}(\emptyset)\right)}^{B}=$

$=\{[0-20),(50-60]\} \cup\{[20-30]\}=\{[0-30],(50-60]\}$

From the previous examples, it follows that argument $C$ is accepted only for the time intervals $\{[0-30],(50-60]\}$.

Given an $E-T A F \Theta=\langle A R, A t t s, A r g A v, A t t A v\rangle$, and an argument $A \in A R$, we will use $A t-P R_{\Theta}(A), A t-S T_{\Theta}(A)$, and $A t-G R_{\Theta}(A)$ to denote the set of intervals on which $A$ is acceptable in $\Theta$ according to at-preferred, at-stable, and atgrounded semantics respectively, using again the skeptical approach where it corresponds. Formally, $A t-P R_{\Theta}(A)=\mathcal{T}_{A}^{P r_{\Theta}}$, where $\left(A, \mathcal{T}_{A}^{P r_{\Theta}}\right)$ is in every atpreferred extension of $\Theta ; A t-S T_{\Theta}(A)=\mathcal{T}_{A}^{S t_{\Theta}}$, where $\left(A, \mathcal{T}_{A}^{S t_{\Theta}}\right)$ is a member of every at-stable extension for the $E-T A F \Theta$; and $A t-G R_{\Theta}(A)=\mathcal{T}_{A}^{G r_{\Theta}}$, where $\left(A, \mathcal{T}_{A}^{G r_{\Theta}}\right)$ is in the at-grounded extension for the $E-T A F \Theta$.

The following property establishes a connection between acceptability in our extended temporal framework $E-T A F$ and acceptability in Dung's frameworks. ${ }^{2}$

Lemma 1. Let $\Theta=\langle A R, A t t s, A r g A v, A t t A v\rangle$ be an $E-T A F$ and let $\alpha \in \mathbb{R}^{+}$ representing a point in time. Let $\Theta_{\alpha}^{\prime}=\left\langle A R_{\alpha}^{\prime}, A t t s_{\alpha}^{\prime}\right\rangle$ be a Dung abstract framework obtained from $\Theta$ in the following way: $A R_{\alpha}^{\prime}=\left\{A \in A R \mid \alpha \in \mathcal{T}_{A}\right\}$ and Atts $_{\alpha}^{\prime}=\left\{(A, B) \in\right.$ Atts $\left.\mid \alpha \in \mathcal{T}_{(A, B)}\right\}$. Let $E$ a collection of t-profiles in $\Theta$, and $E_{\alpha}^{\prime}=\left\{X \mid\left(X, \mathcal{T}_{(X \mid E)}\right) \in E\right.$ and $\left.\alpha \in \mathcal{T}_{(X \mid E)}\right\}$ (thus, $E_{\alpha}^{\prime} \subseteq A R_{\alpha}^{\prime}$ ). It holds that, if $E$ is an at-preferred extension (respectively an at-stable extension or an at-grounded extension) w.r.t. $\Theta$, then $E_{\alpha}^{\prime}$ is a preferred extension (respectively a stable extension or a grounded extension) w.r.t. $\Theta_{\alpha}^{\prime}$.

Intuitively, the $A F \Theta_{\alpha}^{\prime}$ represents a snapshot of the $E$-TAF framework $\Theta$ at the time point $\alpha$, where the arguments and attacks in $\Theta_{\alpha}^{\prime}$ are those that are available at the time point $\alpha$ in $\Theta$. Then, this Lemma states that an at-preferred extension (respectively an at-stable extension or the at-grounded extension) $E$ for $E$-TAF at the time point $\alpha$ coincides with a preferred extension $E_{\alpha}^{\prime}$ (respectively a stable extension or the grounded extension) of $\Theta_{\alpha}^{\prime}$.

The converse of the statement of Lemma 1 does not hold. The fact that $E_{\alpha}$ is an extension with respect to $\Theta_{\alpha}$ does not guarantee that $E$ is an extension with

\footnotetext{
${ }^{2}$ Proofs are included in the Appendix.
} 
respect to $\Theta$, since we only have the certainty that $E$ satisfies the conditions to be an extension in the time point $\alpha$.

Lets see a counterexample. Given a $E-T A F \Theta=\langle A R, A t t s, \operatorname{Arg} A v, A t t A v\rangle$, where:

$$
\begin{aligned}
& -A R=\{A, B\}, \\
& -\operatorname{Atts}=\{(A, B)\}, \\
& -\operatorname{Arg} A v=\{(A,\{[0-40])\} ;(B,\{[30-60]\})\}, \text { and } \\
& -\operatorname{Att} A v=\{((A, B),\{[30-35]\}))\} .
\end{aligned}
$$

If we have a collection of t-profiles $E=\{(A,[0-40]),(B,[30-60])\}$ and assuming that we take into account the time point $\alpha=50$, then we obtain a set of argument $E_{\alpha}=\{B\}$ that is an extension with respect to $\Theta_{\alpha}$, but does not guarantee that $E$ is an extension with respect to $\Theta$. Indeed, $E$ is not a conflictfree collection of t-profiles, since the argument $A$ attacks the arguments $B$ in the time interval $[30-35]$.

\section{Adding Structure to Abstract Argumentation: Structured Argu- mentation Frameworks}

We will now present a form of structured argumentation that maintains a degree of abstraction, but allows to represent the internal structure of an argument. This framework will permit to take into account the information (reasoning steps and evidence) that forms the argument's structure that supports a particular conclusion; these elements are not available in the fully abstract level.

There are several reasons for moving from an abstract argumentation framework to a structured argumentation framework that retains some degree of abstraction, namely: (1) introducing the internal structure of the arguments through a set of reasoning steps and the evidence, gives the possibility of seeing these structured arguments as a generalization of different systems of structured argumentation, such as ABA [16], ASPIC+ [37], Logical Argumentation [13], or DeLP [29], without committing to a particular one; (2) considering the internal structure of arguments allows to consider the aggregation (accrual) of the arguments that support the same conclusion; and, (3) the meta-information associated with each piece in the structure (in this case time availability and reliability), permits to obtain extra information about the argument, by propagating and combining the meta-information associated with the elements of its structure.

Structured Abstract Argumentation Frameworks (SAF) are a simplified version of the Dynamic Argumentation Frameworks (DAF) [41] where argumental structures are conceived as structures standing for trees of smaller abstract entities representing individual reasoning steps, called arguments. In what follows, we will reintroduce the elements of the framework presented in Rotstein et al. [41] necessary for our work. 
The original DAF was designed with the purpose of dealing with dynamics through the consideration of a varying set of evidence. Depending on the contents of the current set of evidence, some arguments will be active and some others will be inactive. Once the set of arguments that are active is ascertained, the situation can be handled as an instance of a Dung's abstract framework; thus, in this sense, these frameworks can be considered as an extension of Dung's frameworks.

To obtain a SAF, we will simplify the DAF formalism by integrating the evidence as part of the set of arguments, as we will mention below. The dynamics of the framework will be handled through the specification of the temporal availability of the arguments, as shown in the next section. Therefore, a SAF $\Gamma$ becomes an enriched $A F$ with the usual components $\langle A R, A t t s\rangle$, but with the addition of structure for arguments, and a preference criterion $\gtrsim$ to determine when an attack is successful becoming part of the Atts relation [42, 2, 3].

The formalism we are introducing for SAF departs from the terminology used in Dung's abstract frameworks as we explain in the following paragraph. The arguments with structure in a SAF are called argumental structures, and the constituent elements are called arguments, where an argument is an abstract entity representing an indivisible reasoning step connecting a set of premisses with a claim; thus, an argument's premises provide backing for the claim. Both, premises and claim, are assumed to be expressed in a language $\mathfrak{L}$ that will remain unspecified and will depend on the domain of application. We will assume sentences in $\mathfrak{L}$ as literals, and use the complement notation to express contradictory literals such as $\alpha$ and $\bar{\alpha}$. The following definition, adapted from [41], formalizes the notion of argument.

Definition 24 (Argument). Given a language $\mathfrak{L}$, an argument $A$ is a reasoning step concluding $\alpha \in \mathfrak{L}$ from a set of premises $\left\{\beta_{1}, \ldots, \beta_{n}\right\} \in 2^{\mathfrak{L}}$, where $\beta_{i} \neq \alpha, \beta_{i} \neq \bar{\alpha}, \beta_{i} \neq \overline{\beta_{j}}$, for $1 \leq i, j \leq n$. Given an argument $A \in$ Args, we will write $\operatorname{cl}(A)$ and $\operatorname{pr}(A)$ to denote its claim and the set of premises involved in it, respectively; also, we will write interface $(A)=\langle\operatorname{pr}(A), \operatorname{cl}(A)\rangle$ to refer to this pair of elements that characterize the argument $A$. Evidence will be considered as arguments where the set of premises is empty, i.e., if $A$ is evidence then $\operatorname{pr}(A)=\emptyset$.

We say that an $\operatorname{argument} B$ supports an argument $A$ if the claim of the $\operatorname{argument} B$ is part of the premises of the argument $A$. Formally:

Definition 25 (Supporting Argument). An argument $B$ is a supporting argument for an argument $A$ iff $\operatorname{cl}(B) \in \operatorname{pr}(A)$, and when $\operatorname{cl}(B)=\beta$ we say that $B$ supports $A$ through $\beta$. Given a set Args of arguments, an argument $A_{i}$ transitively supports an argument $A_{k}$ within Args iff there is a sequence $\left[A_{i}, \ldots, A_{k}\right]$ of arguments in Args where $c l\left(A_{j}\right) \in \operatorname{pr}\left(A_{j+1}\right)$, for every $j$ such that $i \leq j \leq k-1$.

An important relation between arguments is the one that reflects that they might support conflicting conclusions. Formally: 
Definition 26 (Conflict between Arguments). Given a set Args of arguments, the set $\bowtie \subseteq$ Args $\times$ Args denotes a conflict relation over Args, verifying $A_{1} \bowtie A_{2}$ iff $c l\left(A_{1}\right)=\overline{c l}\left(A_{2}\right)$, where $\bar{\alpha}$ denotes the complement of $\alpha$.

Like in DAF, arguments in SAF can be aggregated into argumental structures. These are defined as follows, introducing constraints correspond to sound, nonfallacious argument structures (for full details see [41]).

Definition 27 (Argumental Structure). Given a set Args of arguments and a conflict relation $\bowtie$ defined over Args, an argumental structure in Args for a claim $\alpha$ is a tree of arguments $\mathbb{A}$ verifying:

- Top Argument: The root argument $A_{\text {top }} \in$ Args, called top argument, is such that $\operatorname{cl}\left(A_{\text {top }}\right)=\alpha$, and is noted as top $(\mathbb{A})$;

- Node: $A$ node is an argument $A_{i} \in$ Args such that for each premise $\beta \in \operatorname{pr}\left(A_{i}\right)$ there is exactly one child argument in Args supporting $A_{i}$ through $\beta$;

- Premise Consistency: There are no $\alpha, \beta \in \operatorname{pr}(\mathbb{A})$ such that $\bar{\alpha}=\beta$;

- Consistency: There are no $A, B \in \operatorname{args}(\mathbb{A})$ such that $A \bowtie B$;

- Non-Circularity: No argument $A \in \operatorname{args}(\mathbb{A})$ transitively supports an argument $B \in \operatorname{args}(\mathbb{A})$ if $c l(B) \in \operatorname{pr}(A)$;

- Uniformity: If $A \in \operatorname{args}(\mathbb{A})$ is a child of $B \in \operatorname{args}(\mathbb{A})$ in tree $\mathbb{A}$ and $A$ supports $B$ through $\beta$, then $A$ is a child of every $B_{i} \in \operatorname{args}(\mathbb{A})$ in tree $\mathbb{A}$ such that $\beta \in \operatorname{pr}\left(B_{i}\right)$, supporting $B_{i}$ through $\beta$.

For any argumental structure $\mathbb{A}$, we will write $\operatorname{args}(\mathbb{A})$ to denote the set of arguments in $\mathbb{A}$. Extending the notation, we will also write $\operatorname{cl}(\mathbb{A})=\alpha$ to denote the claim of $\mathbb{A}$, and $\operatorname{pr}(\mathbb{A})$ to denote the set of premises of arguments in $\mathbb{A}$. We will denote $\operatorname{Str}_{(\operatorname{Args}, \bowtie)}$ the set of all argumental structures w.r.t. Args and $\bowtie$.

In Definition 27, the property of consistency invalidates inherently contradictory argumental structures. The requirement of non-circularity avoids taking into consideration structures yielding infinite reasoning chains. Finally, the restriction of uniformity does not allow heterogeneous support for a premise throughout a structure.

Example 6. Let Args be a set of arguments, where Args $=\left\{A_{1}, A_{2}, A_{3}, A_{4}, B_{1}, B_{2}\right.$, $\left.B_{3}, B_{4}, C_{1}, C_{2}\right\}, \bowtie=\left\{\left(A_{1}, B_{1}\right),\left(B_{4}, C_{1}\right)\right\}$, and $\mathbb{A}, \mathbb{B}$, and $\mathbb{C}$ are three argumental structures from $\operatorname{Str}_{(\text {Args }, \bowtie)}$, as depicted in figure 6, where:

$$
\begin{aligned}
& \operatorname{top}(\mathbb{A})=A_{1} \text { and } \operatorname{args}(\mathbb{A})=\left\{A_{1}, A_{2}, A_{3}, A_{4}\right\} \\
& \operatorname{cl}\left(A_{1}\right)=a, \operatorname{pr}\left(A_{1}\right)=\{b, c\}, \operatorname{cl}\left(A_{2}\right)=b, \operatorname{pr}\left(A_{2}\right)=\{d\}, \\
& \operatorname{cl}\left(A_{3}\right)=c, \operatorname{pr}\left(A_{3}\right)=\{f\}, \operatorname{cl}\left(A_{4}\right)=f, \operatorname{pr}\left(A_{4}\right)=\{g, h\} \\
& \operatorname{cl}(\mathbb{A})=a, \operatorname{pr}(\mathbb{A})=\{d, g, h\}
\end{aligned}
$$




$$
\begin{aligned}
& \operatorname{top}(\mathbb{B})=B_{1} \text { and } \operatorname{args}(\mathbb{B})=\left\{B_{1}, B_{2}, B_{3}, B_{4}\right\} \\
& \operatorname{cl}\left(B_{1}\right)=t, \operatorname{pr}\left(B_{1}\right)=\{\sim a, u\}, \operatorname{cl}\left(B_{2}\right)=\sim a, \operatorname{pr}\left(B_{2}\right)=\{v\}, \\
& \operatorname{cl}\left(B_{3}\right)=u, \operatorname{pr}\left(B_{3}\right)=\{p\}, \operatorname{cl}\left(B_{4}\right)=p, \operatorname{pr}\left(B_{4}\right)=\{x, y\} \\
& \operatorname{cl}(\mathbb{B})=t, \operatorname{pr}(\mathbb{B})=\{v, x, y\} \\
& \operatorname{top}(\mathbb{C})=C_{1} \text { and } \operatorname{args}(\mathbb{C})=\left\{C_{1}, C_{2}\right\} \\
& \operatorname{cl}\left(C_{1}\right)=\sim t, \operatorname{pr}\left(C_{1}\right)=\{q\}, \operatorname{cl}\left(C_{2}\right)=q, \operatorname{pr}\left(C_{2}\right)=\{r\}, \\
& \operatorname{cl}(\mathbb{C})=\sim t, \operatorname{pr}(\mathbb{C})=\{r\}
\end{aligned}
$$

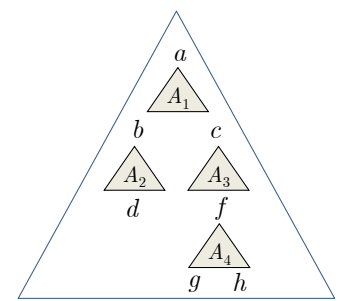

$\mathbb{A}$

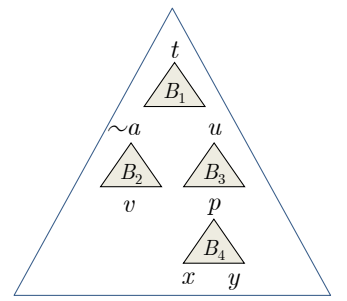

$\mathbb{B}$

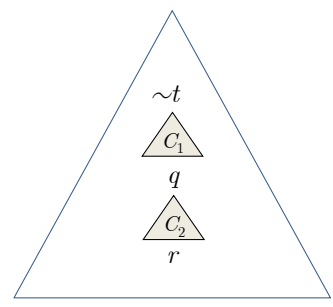

$\mathbb{C}$

Figure 6: Representation of a Argumental Structures (Example 6)

It is important to stress that, within an argumental structure, given the Uniformity requirement, a premise of an argument cannot be supported by different arguments. For the sake of simplicity, in the sequel we will refer to argumental structures just as "structures".

Not any subset of the set of arguments of a given structure is a substructure of it; that is, the arguments that are part of a substructure they should form a structure themselves following the requirements of Definition 27. Formally:

Definition 28 (Substructures). Let Args be a set of arguments and $\mathbb{A}, \mathbb{A}^{\prime} \subseteq$ Args be two argumental structures. We will say that $\mathbb{A}^{\prime}$ is an argumental substructure of $\mathbb{A}$ iff $\operatorname{args}\left(\mathbb{A}^{\prime}\right) \subseteq \operatorname{args}(\mathbb{A})$.

The defeat relation in SAF, called attack in abstract frameworks, can be obtained through the application of a preference relation over the structures forming conflicting pairs (called attacks in SAF). When adding a preference criterion over conflicts, this relationship can be refined into a defeat relation between arguments.

Note that the attack relation in a Dung's abstract argumentation framework assumes that the attack is always successful becoming a defeat in the terminology used in a SAF; meanwhile, in a SAF, the conflict has to be resolved using a preference criterion, so that it becomes a defeat. We will now introduce successively Conflict, Preference, and Defeat. Formally: 
Definition 29 (Conflict between structures). Let $\operatorname{Str}_{(\text {Args }, \bowtie)}$ a set of structures, and let $\mathbb{A}, \mathbb{A}^{\prime} \in \operatorname{Str}_{\left(\text {Args }_{,} \bowtie\right)}$ be two structures. We will say that $\mathbb{A}$ is in conflict with $\mathbb{B}$, denoted as $\mathbb{A} \asymp \mathbb{B}$, iff there is an argumental substructure $\mathbb{B}^{\prime}$ of $\mathbb{B}$ such that top $(\mathbb{A}) \bowtie \operatorname{top}\left(\mathbb{B}^{\prime}\right)$. The structure $\mathbb{B}^{\prime}$ is called the disagreement substructure.

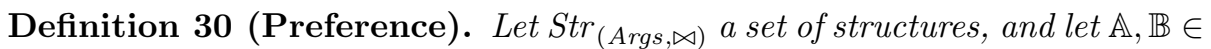
$\operatorname{Str}_{\left(\operatorname{Args}_{,} \bowtie\right)}$ be two structures in $\operatorname{Str}_{(\operatorname{Args}, \bowtie)}$. Given a preference relation ' $\gtrsim$ ' defined over $\operatorname{Str}_{(\text {Args, } \bowtie)}$, we will say that $\mathbb{A}$ is at least as preferred as $\mathbb{B}$ when the relation is satisfied.

This relation usually codifies semantic notions of argument comparison. For a syntactic criterion that could be adapted to SAF see [42, 43].

Definition 31 (Defeat between structures). Let $\operatorname{Str}_{(A r g s, \bowtie)}$ a set of structures, and let $\mathbb{A}, \mathbb{B} \in \operatorname{Str}_{\left(A_{r g s}, \bowtie\right)}$ be two structures in $\operatorname{Str}_{(\operatorname{Args}, \bowtie)}$. We will say that $\mathbb{A}$ defeats $\mathbb{B}$, iff $\mathbb{A} \asymp \mathbb{B}$ with disagreement structure $\mathbb{B}^{\prime}$ of $\mathbb{A}$ such that $\mathbb{A} \gtrsim \mathbb{B}^{\prime}$.

The following example illustrates the definitions just introduced.

Example 7. In Example $6, \mathbb{A}$ is in conflict with $\mathbb{B}(\mathbb{A} \asymp \mathbb{B})$, since $\mathbb{B}$ has a substructure $\mathbb{B}^{\prime}$ that contains the argument $B_{2}$, and since as a and $\sim a$ are contradictory $\mathbb{A} \bowtie \mathbb{B}^{\prime}$ (Figure 7 shows the chosen $\mathbb{B}^{\prime}$ inside a dotted triangle). If the preference criterion sanctions that $\mathbb{A} \gtrsim \mathbb{B}^{\prime}$, the result is that $\mathbb{A}$ defeats $\mathbb{B}$.

Also in Example $6, \mathbb{B}$ is in conflict with $\mathbb{C}(\mathbb{B} \asymp \mathbb{C})$, since $\mathbb{C}$ is trivially a substructure of itself, and considering $\mathbb{C}$ as the $\mathbb{B}^{\prime}$ of the definition above(again, Figure 7 shows the required $\mathbb{B}^{\prime}$ inside a dotted triangle) we see that $\mathbb{B} \bowtie \mathbb{C}$ assuming logical contradiction between $t$ and $\sim t$. Furthermore, if the preference criterion determines that $\mathbb{B} \gtrsim \mathbb{C}$, it results that $\mathbb{B}$ defeats $\mathbb{C}$.

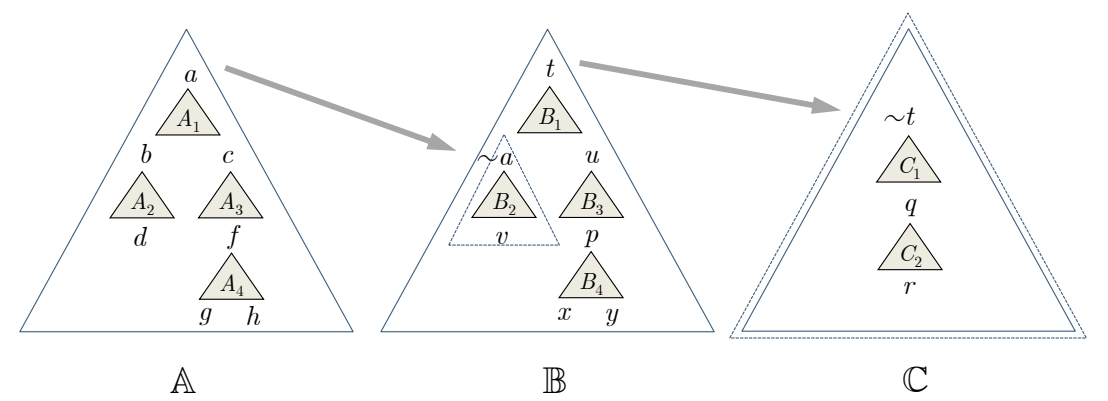

Figure 7: Attack and Defeat between Argumental Structures (Example 7)

Definition 32 (Structured Argumentation Framework). A Structured Argumentation Framework $\Gamma$ is a tuple $\langle$ Args, $\bowtie, \gtrsim\rangle$, where Args is a set of arguments, $\bowtie$ is a conflict relation, and $\gtrsim$ is a preference criterion defined over the set of all argumental structures $\operatorname{Str}_{(\operatorname{Args}, \bowtie)}$. 
All the semantic notions, such as acceptability and the usual argumentation semantics, can be obtained for SAF just by instantiating Dung's abstract framework $A F=\langle A R, A t t s\rangle$ with the set of all argumental structures $\operatorname{Str}_{\left(\operatorname{Args}_{,} \bowtie\right)}$ as the set $A R$ and the defeat relation among structures as the Atts relation. We will not explore this issue any further in this paper.

\section{6. $E-T A F^{*}: E-T A F$ over Argumental Structures}

Many applications of argumentation require the explicit treatment of time; usually, temporal information is not directly associated with arguments, but instead it is attached to the basic pieces of knowledge (e.g., claims, rules, premises) from which arguments are built. Other interesting features of arguments, such as valuation, may also vary in time, causing the availability of attacks to change dynamically. As with temporal information, valuation can be associated with individual components of an argument, and propagated through a suitable valuation function as information attached to the whole argument [19].

We move in that direction by replacing $E$-TAF abstract arguments with SAF argumental structures. As we will see next, this expanded framework, referred to as $E-T A F^{*}$, is expressive enough to capture temporal availability and the notion of valuation varying over time as well, associated with individual steps (called arguments in SAF) from which the argumental structures are built. Argumental structures' availability and valuation are obtained by integrating the corresponding information attached to the arguments composing them.

The instantiation of E-TAF's arguments with SAF's argumental structures gives us the ability of studying the temporal availability and valuation of argumental structures as a function of its internal components. Thus, we will be able to determine how each component affects these features of an argumental structure. Temporal availability and valuation factors are associated with SAF arguments through the formal notion of $\tau^{*}$-argument, as we define below.

Definition 33 ( $\tau^{*}$-argument). Let $\Gamma=\langle$ Args, $\bowtie, \gtrsim\rangle$ be a $S A F$, called the subjacent structured argument framework. We say $\left\langle A, \mathcal{T}_{A}, v_{A}\right\rangle$ is a $\tau^{*}$-argument for the claim $\alpha$ in $\Gamma$ whenever:

i) $A \in$ Args is an argument in $\Gamma$ (see Definition 24) such that $c l(A)=\alpha$.

ii) $\mathcal{T}_{A} \subseteq \mathbb{R}^{+}$is the time interval set in which $A$ is available.

iii) $v_{A}: \mathbb{R}^{+} \longrightarrow[0,1]$ is a function that expresses the valuation of $A$ over time.

The set of $\tau^{*}$-arguments over $\Gamma$ will be denoted $\operatorname{Args}_{\Gamma}$, and $\Gamma$ will be referred to as the subjacent $S A F$.

Definition 34 (Subjacent Argument(s)). Let $\Gamma=\langle$ Args, $\bowtie, \gtrsim\rangle$ be a SAF. Given a $\tau^{*}$-argument $\mathrm{A}=\left\langle A, \mathcal{T}_{A}, v_{A}\right\rangle$ we will define a function subj $(\cdot)$ that will return the argument in $\Gamma$ involved in the $\tau^{*}$-argument, i.e., subj $\left(\left\langle A, \mathcal{T}_{A}, v_{A}\right\rangle\right)=$ A. Thus, $\operatorname{cl}\left(\operatorname{subj}\left(\left\langle A, \mathcal{T}_{A}, v_{A}\right\rangle\right)\right)=\operatorname{cl}(A)$, and $\operatorname{pr}\left(\operatorname{subj}\left(\left\langle A, \mathcal{T}_{A}, v_{A}\right\rangle\right)\right)=\operatorname{pr}(A)$ will be the claim and the set of premises of $\left\langle A, \mathcal{T}_{A}, v_{A}\right\rangle$. 
As in SAF, arguments may be in conflict, however in $E-T A F^{*}$ conflict depends on the availability of the arguments involved. Therefore, two $\tau^{*}$-arguments supporting contradictory information will be in conflict only when their time availability overlaps, i.e., over the intersection of the temporal intervals in which they are available; that is, we will restrict the conflict relation $\bowtie$ to the temporal intervals where both $\tau^{*}$-arguments are available simultaneously.

Definition 35 (Conflict between $\tau^{*}$-arguments). Let $\Gamma=\langle$ Args, $\bowtie, \gtrsim\rangle$ be a SAF. Given a set $\operatorname{Args}_{\Gamma}$ of $\tau^{*}$-arguments, the relation $\bowtie \subseteq \operatorname{Args}_{\Gamma} \times \operatorname{Args}_{\Gamma}$ denotes a conflict relation over Args, propagating $\bowtie$, the conflict relation of the subjacent $\Gamma:\left\langle A, \mathcal{T}_{A}, v_{A}\right\rangle \bowtie\left\langle B, \mathcal{T}_{B}, v_{B}\right\rangle$ iff $\operatorname{subj}\left(\left\langle A, \mathcal{T}_{A}, v_{A}\right\rangle\right) \bowtie \operatorname{subj}\left(\left\langle B, \mathcal{T}_{B}, v_{B}\right\rangle\right)$, (i.e., $A \bowtie B$ ), where $\mathcal{T}_{A} \cap \mathcal{T}_{B} \neq \emptyset$, and $\bowtie$ is conflict relation in the subjacent framework $\Gamma$.

Once the definition of $\tau^{*}$-argument is introduced as a reasoning step labeled with temporal availability in a time interval set $\mathcal{T}$, and the valuation factor over $\mathcal{T}$ is defined, we will introduce the notion of $\tau^{*}$-structure in $E$-TAF $F^{*}$. Informally, a $\tau^{*}$-structure $\mathbb{A}$ is a tentative proof (as it relies to some extent on valuation that varies over time) from a consistent set of $\tau^{*}$-arguments, supporting a given conclusion $Q$, and specifying its valuation degree over time.

Definition 36 ( $\tau^{*}$-structure). Let $\Gamma=\langle$ Args, $\bowtie, \gtrsim\rangle$ be a $S A F$. Let $\operatorname{Args}_{\Gamma}$ be a set of $\tau^{*}$-arguments and let $\bowtie$ be a conflict relation over $\operatorname{Args}_{\Gamma}$. We say that $\left(\mathbb{A}, \mathcal{T}_{\mathbb{A}}, v_{\mathbb{A}}\right)$ is a $\tau^{*}$-argumental structure, or just a $\tau^{*}$-structure, supporting a conclusion $\alpha$, iff

$-\operatorname{subj}(\mathbb{A})$ is an argumental structure over $\Gamma$, and $\operatorname{cl}(\operatorname{top}(\operatorname{subj}(\mathbb{A})))=\alpha$;

- $\mathcal{T}_{\mathbb{A}}=\bigcap_{i=1}^{n} \mathcal{T}_{A_{i}}$ where $\mathcal{T}_{A_{i}}$ is the time interval for $\tau^{*}$-argument that corresponds to $\left\langle A_{i}, \mathcal{T}_{A_{i}}, v_{A_{i}}\right\rangle \in \mathbb{A} ;$ if $\mathcal{T}_{\mathbb{A}}=\emptyset$ we say that the $\mathbb{A}$ is inactive.

- $v_{\mathbb{A}}: \mathbb{R}^{+} \longrightarrow[0,1]$, such that $v_{\mathbb{A}}(t)=\min \left\{v_{A_{i}}(t) \mid 1 \leq i \leq n\right\}$, where $v_{A_{i}}(t)$ corresponds to the $\tau^{*}$-argument $\left\langle A_{i}, \mathcal{T}_{A_{i}}, v_{A_{i}}\right\rangle \in \mathbb{A}$.

The set of all $\tau^{*}$-structures w.r.t. $\operatorname{Args}_{\Gamma}$ and $\bowtie$ is denoted as $\operatorname{Str}_{(\operatorname{Args}, \bowtie)}$. We extend the notation $\operatorname{subj}(\cdot)$ to a set $\mathbb{A} \subseteq \operatorname{Str}_{(\operatorname{Args}, \bowtie)}$ as $\operatorname{subj}(\mathbb{A})=\{\operatorname{subj}(\overline{\mathrm{A}}) \mid \mathrm{A} \in \mathbb{A}\}$, notice that $\operatorname{subj}(\mathbb{A})$ is a subset of Args from the subjacent $S A F \Gamma$. When no confusion is possible, we will also extend $\operatorname{cl}(\cdot), \operatorname{pr}(\cdot)$, and interface $(\cdot)$ to $\operatorname{cl}(\mathbb{A}), \operatorname{pr}(\mathbb{A})$, and interface $(\mathbb{A})$ to refer to the claim, the premises, and the interface of $\mathbb{A}$.

Definition $37\left(\tau^{*}\right.$-substructure). Let $\operatorname{Str}_{(\operatorname{Args}, \unrhd)}$ be a set of $\tau^{*}$-arguments,

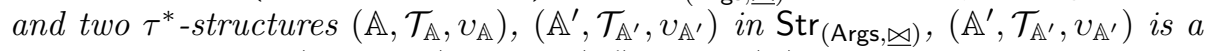
$\tau^{*}$-substructure of $\left(\mathbb{A}, \mathcal{T}_{\mathbb{A}}, v_{\mathbb{A}}\right)$ iff $\operatorname{subj}\left(\mathbb{A}^{\prime}\right) \subseteq \operatorname{subj}(\mathbb{A})$. We will use the notation $\left(\mathbb{A}^{\prime}, \mathcal{T}_{\mathbb{A}^{\prime}}, v_{\mathbb{A}^{\prime}}\right) \subseteq\left(\mathbb{A}, \mathcal{T}_{\mathbb{A}}, v_{\mathbb{A}}\right)$ to indicate this relation when no confusion is possible.

The property of the $\tau^{*}$-substructure relation expressed in the proposition below is obvious from the definition of $\tau^{*}$-structure. 
Proposition 5. Let $\operatorname{Str}(\operatorname{Args}, \bowtie)_{1}$ be a set of $\tau^{*}$-arguments, given two $\tau^{*}$-arguments $\left(\mathbb{A}, \mathcal{T}_{\mathbb{A}}, v_{\mathbb{A}}\right),\left(\mathbb{A}^{\prime}, \mathcal{T}_{\mathbb{A}^{\prime}}, v_{\mathbb{A}^{\prime}}\right)$ in $\operatorname{Str}\left(\operatorname{Args}_{\mathbb{1}}, \mathbb{}\right)$, where $\left(\mathbb{A}^{\prime}, \mathcal{T}_{\mathbb{A}^{\prime}}, v_{\mathbb{A}^{\prime}}\right)$ is a $\tau^{*}$-substructure of $\left(\mathbb{A}, \mathcal{T}_{\mathbb{A}}, v_{\mathbb{A}}\right)$ then $\mathcal{T}_{\mathbb{A}} \subseteq \mathcal{T}_{\mathbb{A}^{\prime}}$ and $v_{\mathbb{A}} \leq v_{\mathbb{A}^{\prime}}$.

Finally, the definition of attack relation is analogous to that in SAF.

Definition 38 (Conflict between $\tau^{*}$-structures). Given $\operatorname{Str}(\operatorname{Args}, \bowtie)$, and $\tau^{*}$-structures $\left(\mathbb{A}, \mathcal{T}_{\mathbb{A}}, v_{\mathbb{A}}\right),\left(\mathbb{B}, \mathcal{T}_{\mathbb{B}}, v_{\mathbb{B}}\right) \in \operatorname{Str}_{(\text {Args, } ₫)}$, such that $\mathcal{T}_{\mathbb{A}} \cap \mathcal{T}_{\mathbb{B}} \neq \emptyset$, then $\left(\mathbb{A}, \mathcal{T}_{\mathbb{A}}, v_{\mathbb{A}}\right)$ is in conflict with $\left(\mathbb{B}, \mathcal{T}_{\mathbb{B}}, v_{\mathbb{B}}\right)$, denoted $\left(\mathbb{A}, \mathcal{T}_{\mathbb{A}}, v_{\mathbb{A}}\right) \asymp\left(\mathbb{B}, \mathcal{T}_{\mathbb{B}}, v_{\mathbb{B}}\right)$, iff there exists a $\tau^{*}$-substructure $\left(\mathbb{B}^{\prime}, \mathcal{T}_{\mathbb{B}^{\prime}}, v_{\mathbb{B}^{\prime}}\right)$ of $\left(\mathbb{B}, \mathcal{T}_{\mathbb{B}}, v_{\mathbb{B}}\right)$ such that top $(\mathbb{A}) \bowtie$ top $\left(\mathbb{B}^{\prime}\right)$. Furthermore, the $\tau^{*}$-substructure $\left(\mathbb{B}^{\prime}, \mathcal{T}_{\mathbb{B}^{\prime}}, v_{\mathbb{B}^{\prime}}\right)$ is called the disagreement $\tau^{*}$-substructure.

To formalize the notion of acceptability of a $\tau^{*}$-structure in $E-T A F^{*}$, we have to consider that $E-T A F^{*}$ is a parameterized version of $E-T A F$ w.r.t. a SAF $\Gamma$ in which we rely on compositional features for capturing time availability and valuation of arguments. As a consequence, we can use the same 4-tuple characterizing $E-T A F$ in order to formalize $E$ - $T A F^{*}$, provided that a SAF characterization is also given.

Definition $39\left(E-T A F^{*}\right)$. Let $\Gamma=\langle A r g s, \bowtie, \gtrsim\rangle$ be a $S A F$. Then an $E-T A F^{*}$ is a tuple $\Psi=\left\langle\operatorname{Str}_{(\operatorname{Args}, \bowtie)}, \asymp\right.$, AttAv, $\left.\geq\right\rangle$ where:

i) $\operatorname{Str}_{(\operatorname{Args}, \bowtie)}$ is a set of $\tau^{*}$-structures.

ii) $\asymp$ is a conflict relation between $\tau^{*}$-structures;

iv) Given $\left(\mathbb{A}, \mathcal{T}_{\mathbb{A}}, v_{\mathbb{A}}\right) \asymp\left(\mathbb{B}, \mathcal{T}_{\mathbb{B}}, v_{\mathbb{B}}\right)$, with the $\tau^{*}$-substructure $\left(\mathbb{B}^{\prime}, \mathcal{T}_{\mathbb{B}^{\prime}}, v_{\mathbb{B}^{\prime}}\right)$ as the disagreement $\tau^{*}$-structure of $\left(\mathbb{B}, \mathcal{T}_{\mathbb{B}}, v_{\mathbb{B}}\right)$ with $\left(\mathbb{A}, \mathcal{T}_{\mathbb{A}}, v_{\mathbb{A}}\right)$, then the availability of the attack is:

$$
\operatorname{AttAv}\left(\left(\left(\mathbb{A}, \mathcal{T}_{\mathbb{A}}, v_{\mathbb{A}}\right),\left(\mathbb{B}, \mathcal{T}_{\mathbb{B}}, v_{\mathbb{B}}\right)\right)\right)={ }_{\operatorname{def}} \mathcal{T}_{\mathbb{A}} \cap \mathcal{T}_{\mathbb{B}^{\prime}}
$$

We will simplify the notation using $\mathcal{T}_{(\mathbb{A}, \mathbb{B})}$ instead of the more complex expression $\operatorname{Att} A v\left(\left(\left(\mathbb{A}, \mathcal{T}_{\mathbb{A}}, v_{\mathbb{A}}\right),\left(\mathbb{B}, \mathcal{T}_{\mathbb{B}}, v_{\mathbb{B}}\right)\right)\right)$. Notice that an attack from $\left(\mathbb{A}, \mathcal{T}_{\mathbb{A}}, v_{\mathbb{A}}\right)$ to $\left(\mathbb{B}, \mathcal{T}_{\mathbb{B}}, v_{\mathbb{B}}\right)$ is available only in the time intervals where the valuation of $\left(\mathbb{A}, \mathcal{T}_{\mathbb{A}}, v_{\mathbb{A}}\right)$ is greater or equal than the valuation of $\left(\mathbb{B}, \mathcal{T}_{\mathbb{B}}, v_{\mathbb{B}}\right)$. This is reflected in the following definition.

Definition 40 (Defeat between $\tau^{*}$-structures). Given the E-TAF* $\Psi$, defined as $\Psi=\left\langle\operatorname{Str}_{(\operatorname{Args}, \unrhd)}, \succeq, \operatorname{Att} A v, \geq\right\rangle$, and $\left(\mathbb{A}, \mathcal{T}_{\mathbb{A}}, v_{\mathbb{A}}\right),\left(\mathbb{B}, \mathcal{T}_{\mathbb{B}}, v_{\mathbb{B}}\right) \in \operatorname{Str}_{(\operatorname{Args}, \unrhd)}$ wich are in conflict having $\left(\mathbb{B}^{\prime}, \mathcal{T}_{\mathbb{B}^{\prime}}, v_{\mathbb{B}^{\prime}}\right) \subseteq\left(\mathbb{B}, \mathcal{T}_{\mathbb{B}}, v_{\mathbb{B}}\right)$ as the disagreement substructure, we say that $\left(\mathbb{A}, \mathcal{T}_{\mathbb{A}}, v_{\mathbb{A}}\right)$ defeats $\left(\mathbb{B}, \mathcal{T}_{\mathbb{B}}, v_{\mathbb{B}}\right)$ in the time interval $\mathcal{T}_{\left(\mathbb{A}, \mathbb{B}^{\prime}\right)}$, if $v_{\mathbb{A}} \geq v_{\mathbb{B}^{\prime}}$ over that time interval.

Clearly, the notions of defense and acceptability for $E-T A F^{*}$ can be defined in a similar way as was defined for $E$-TAF, taking into account the interactions between $\tau^{*}$-structures and the reliability function associated with each of them. 
Definition 41 (Defense of $\left(\mathbb{A}, \mathcal{T}_{\mathbb{A}}, v_{\mathbb{A}}\right)$ from $\left(\mathbb{B}, \mathcal{T}_{\mathbb{B}}, v_{\mathbb{B}}\right)$ by $\left.S\right)$. Let $\Psi$ be an $E-T A F^{*}$ defined as $\Psi=\left\langle\operatorname{Str}_{(\operatorname{Args}, \bowtie)}, \asymp, \operatorname{Att} A v, \geq\right\rangle$, let $\left(\mathbb{A}, \mathcal{T}_{\mathbb{A}}, v_{\mathbb{A}}\right)$ and $\left(\mathbb{B}, \mathcal{T}_{\mathbb{B}}, v_{\mathbb{B}}\right)$ be two $\tau^{*}$-structures where $\left(\mathbb{A}, \mathcal{T}_{\mathbb{A}}, v_{\mathbb{A}}\right) \asymp\left(\mathbb{B}, \mathcal{T}_{\mathbb{B}}, v_{\mathbb{B}}\right)$, and let $S$ be an at-conflictfree set of $\tau^{*}$-structures such that $S \subseteq \operatorname{Str}_{(\operatorname{Args}, \bowtie)}$. The defense t-profile of $\left(\mathbb{A}, \mathcal{T}_{\mathbb{A}}, v_{\mathbb{A}}\right)$ from $\left(\mathbb{B}, \mathcal{T}_{\mathbb{B}}, v_{\mathbb{B}}\right)$ w.r.t. $S$, denoted as $\mathcal{T}_{(\mathbb{A} \mid S)}^{\mathbb{B}}$ is defined as follows:

$$
\mathcal{T}_{(\mathbb{A} \mid S)}^{\mathbb{B}}={ }_{\text {def }} \bigcup_{\left(\mathbb{C}, \mathcal{T}_{\mathbb{C}}, v_{\mathbb{C}}\right) \in\left\{\left(\mathbb{X}, \mathcal{T}_{\mathbb{X}}, v_{\mathbb{X}}\right) \in S, \mid \mathcal{T}_{(\mathbb{X}, \mathbb{A})}\right\}}\left(\mathcal{T}_{(\mathbb{B}, \mathbb{A})} \cap \mathcal{T}_{(\mathbb{C}, \mathbb{B})} \cap \mathcal{T}_{\mathbb{C}}\right)
$$

Definition 42 (Acceptable $t$-profile of $\left(\mathbb{A}, \mathcal{T}_{\mathbb{A}}, v_{\mathbb{A}}\right)$ w.r.t. $S$ ). Let $\Psi$ be an $E$-TAF $F^{*}$ defined as $\Psi=\left\langle\operatorname{Str}_{(\operatorname{Args}, \bowtie)}, \asymp\right.$, AttAv, $\left.\geq\right\rangle$, let $S$ be a set of $\tau^{*}$-structures such that $S \subseteq \operatorname{Str}_{(\operatorname{Args}, \bowtie)}$. The acceptable t-profile for $\left(\mathbb{A}, \mathcal{T}_{\mathbb{A}}, v_{\mathbb{A}}\right)$ w.r.t. to $S$, denoted as $\mathcal{T}_{(\mathbb{A} \mid S)}$ is defined as follows:

$$
\mathcal{T}_{(\mathbb{A} \mid S)}={ }_{\operatorname{def}} \bigcap_{\left(\mathbb{B}, \mathcal{T}_{\mathbb{B}}, v_{\mathbb{B}}\right) \in\left\{\left(\mathbb{X}, \mathcal{T}_{\mathbb{X}}, v_{\mathbb{X}}\right) \mid \mathcal{T}_{(\mathbb{X}, \mathbb{A})}\right\}}\left(\mathcal{T}_{\mathbb{A}} \backslash \mathcal{T}_{(\mathbb{B}, \mathbb{A})}\right) \cup \mathcal{T}_{(\mathbb{A} \mid S)}^{\mathbb{B}}
$$

Where $\mathcal{T}_{(\mathbb{A} \mid S)}^{\mathbb{B}}$ is the time interval where $\left(\mathbb{A}, \mathcal{T}_{\mathbb{A}}, v_{\mathbb{A}}\right)$ is defended by $S$ of its attacker $\left(\mathbb{B}, \mathcal{T}_{\mathbb{B}}, v_{\mathbb{B}}\right)$.

Thus, this definition reflects that the intersection of all time intervals in which an argument is defended from each of its attackers by the set $S$, is the time interval where the argument is available and it is acceptable with respect to $S$. Now using the elements from the above definition. The at-preferred, at-stable, and at-grounded semantics for $E-T A F^{*}$ can be characterized in a similar way as done for $E-T A F$.

As illustrated by the example in Fig. 5, it is possible for two otherwise conflicting arguments in an $E$-TAF to be accepted with respect to at-grounded semantics over different time intervals where the conflict does not appear; for instance, this happens when the attack relation is available in a proper subset of the time where the attacked argument is available. In practice, this situation may arise when the existence of the conflict between arguments depends on time, as was the case in the example represented in Fig. 5. For instance, two arguments supporting, respectively, traveling in vacations and buying a new car may be in conflict in certain time intervals where the person cannot afford both expenditures; but, the conflict does not exist in other intervals where the person has the money for both things (i.e., the attack is not available in these intervals).

However, this situation cannot happen when the availability of attacks varying over time is consequence of valuation (or more generally, strength) of arguments varying over time, as is the case with $E$ - TAF*. Recalling that the notation $A t-S T_{\Psi}\left(\left(\mathbb{A}, \mathcal{T}_{\mathbb{A}}, v_{\mathbb{A}}\right)\right), A t-P R_{\Psi}\left(\left(\mathbb{A}, \mathcal{T}_{\mathbb{A}}, v_{\mathbb{A}}\right)\right)$, and $A t-G R_{\Psi}\left(\left(\mathbb{A}, \mathcal{T}_{\mathbb{A}}, v_{\mathbb{A}}\right)\right)$, is used to denote the set of time intervals on which $\left(\mathbb{A}, \mathcal{T}_{\mathbb{A}}, v_{\mathbb{A}}\right)$ is acceptable according to at-stable, at-preferred, and at-grounded semantics respectively in $\Psi$, the following lemma establishes formally that two $\tau^{*}$-structure cannot coincide in time if both belong to the same extension in a given semantics. 
Proposition 6. Given the $E-T A F^{*} \Psi=\left\langle\operatorname{Str}_{\left(\operatorname{Args}_{,} \bowtie\right)}, \asymp, A t t A v, \geq\right\rangle$ and the two $\tau^{*}$-structures $\left(\mathbb{A}, \mathcal{T}_{\mathbb{A}}, v_{\mathbb{A}}\right),\left(\mathbb{B}, \mathcal{T}_{\mathbb{B}}, v_{\mathbb{B}}\right) \in \operatorname{Str}_{(\operatorname{Args}, \bowtie)}$. If the $\tau^{*}$-structure $\left(\mathbb{A}, \mathcal{T}_{\mathbb{A}}, v_{\mathbb{A}}\right)$ defeats $\left(\mathbb{B}^{\prime}, \mathcal{T}_{\mathbb{B}^{\prime}}, v_{\mathbb{B}^{\prime}}\right)$ over the time interval $\mathcal{T}_{\mathbb{A}} \cap \mathcal{T}_{\mathbb{B}^{\prime}}$, where $\left(\mathbb{B}^{\prime}, \mathcal{T}_{\mathbb{B}^{\prime}}, v_{\mathbb{B}^{\prime}}\right)$ is a $\tau^{*}$ substructure of $\left(\mathbb{B}, \mathcal{T}_{\mathbb{B}}, v_{\mathbb{B}}\right)$ then it holds that:

- $\mathcal{T}_{\mathbb{A}}^{P r_{\Psi}} \cap \mathcal{T}_{\mathbb{B}}^{P r_{\Psi}}=\emptyset$,

- $\mathcal{T}_{\mathbb{A}}^{S t_{\Psi}} \cap \mathcal{T}_{\mathbb{B}}^{S t_{\Psi}}=\emptyset$, and

- $\mathcal{T}_{\mathbb{A}}^{G r_{\Psi}} \cap \mathcal{T}_{\mathbb{B}}^{G r_{\Psi}}=\emptyset$.

The key of the proof is to consider the disagreement $\tau^{*}$-substructure $\left(\mathbb{B}^{\prime}, \mathcal{T}_{\mathbb{B}^{\prime}}, v_{\mathbb{B}^{\prime}}\right)$ of $\left(\mathbb{B}, \mathcal{T}_{\mathbb{B}}, v_{\mathbb{B}}\right)$. Even if the attack of $\left(\mathbb{A}, \mathcal{T}_{\mathbb{A}}, v_{\mathbb{A}}\right)$ against $\left(\mathbb{B}, \mathcal{T}_{\mathbb{B}}, v_{\mathbb{B}}\right)$ is not available in certain time intervals, we can indeed ensure that the arguments are not simultaneously accepted, since in those intervals it will be an available attack from the disagreement $\tau^{*}$-substructure $\left(\mathbb{B}^{\prime}, \mathcal{T}_{\mathbb{B}^{\prime}}, v_{\mathbb{B}^{\prime}}\right)$ back to $\left(\mathbb{A}, \mathcal{T}_{\mathbb{A}}, v_{\mathbb{A}}\right)$. Notice that if the attack of $\left(\mathbb{A}, \mathcal{T}_{\mathbb{A}}, v_{\mathbb{A}}\right)$ against $\left(\mathbb{B}, \mathcal{T}_{\mathbb{B}}, v_{\mathbb{B}}\right)$ is not available then it means that the disagreement $\tau^{*}$-substructure $\left(\mathbb{B}^{\prime}, \mathcal{T}_{\mathbb{B}^{\prime}}, v_{\mathbb{B}^{\prime}}\right)$ is more reliable than $\left(\mathbb{A}, \mathcal{T}_{\mathbb{A}}, v_{\mathbb{A}}\right)$.

It can be shown that the at-stable (respectively at-preferred or at-grounded) intervals set associated with a $\tau^{*}$-structure is a subset of the at-stable (respectively at-preferred or at-grounded) intervals set of any of its $\tau^{*}$-substructures. Formally:

Proposition 7. Given the $E-T A F^{*} \Psi=\left\langle\operatorname{Str}_{(\operatorname{Args}, \bowtie)}, \asymp, A t t A v, \geq\right\rangle$ and the two

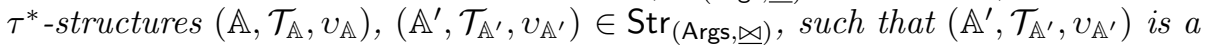
$\tau^{*}$-substructure of $\left(\mathbb{A}, \mathcal{T}_{\mathbb{A}}, v_{\mathbb{A}}\right)$. Then:

- $\mathcal{T}_{\mathbb{A}^{\prime}}^{P r_{\Psi}} \supseteq \mathcal{T}_{\mathbb{A}}^{P r_{\Psi}}$,

- $\mathcal{T}_{\mathbb{A}^{\prime}}^{S t_{\Psi}} \supseteq \mathcal{T}_{\mathbb{A}}^{S t_{\Psi}}$, and

- $\mathcal{T}_{\mathbb{A}^{\prime}}^{G r_{\Psi}} \supseteq \mathcal{T}_{\mathbb{A}}^{G r_{\Psi}}$.

In the next example we show how the different elements introduced previously can be used to analyze acceptability in $E-T A F^{*}$.

Example 8. Let us consider the following set of $\tau^{*}$-arguments:

$$
\left\{\begin{array}{cc}
\left\langle A_{1},\{[0-60]\}, v_{1}\right\rangle & \left\langle B_{2},\{[0-50]\}, v_{6}\right\rangle \\
\left\langle A_{2},\{[0-80]\}, v_{2}\right\rangle & \left\langle B_{3},\{[10-90]\}, v_{7}\right\rangle \\
\left\langle A_{3},\{[0-30]\}, v_{3}\right\rangle & \left\langle B_{4},\{[0-80]\}, v_{8}\right\rangle \\
\left\langle A_{4},\{[0-40]\}, v_{4}\right\rangle & \left\langle C_{1},\{[0-90]\}, v_{9}\right\rangle \\
\left\langle B_{1},\{[0-100]\}, v_{5}\right\rangle & \left\langle C_{2},\{[0-60]\}, v_{10}\right\rangle
\end{array}\right\}
$$

where the valuation functions are defined below: 


$$
\begin{array}{ll}
v_{1}(\alpha)=.5 & v_{6}(\alpha)= \begin{cases}.7 & \alpha<15 \\
.3 & 15 \leq \alpha \leq 40 \\
.9 & \alpha>40\end{cases} \\
v_{2}(\alpha)=1 & v_{7}(\alpha)= \begin{cases}1 & \alpha<50 \\
.8 & \alpha \geqslant 50\end{cases} \\
v_{3}(\alpha)=.9 & v_{8}(\alpha)=.7 \\
v_{4}(\alpha)=.7 & v_{9}(\alpha)= \begin{cases}1 & \alpha<20 \\
.1 & 20 \leq \alpha \leq 60 \\
.7 & \alpha>60 \\
v_{5}(\alpha)=1 & v_{10}(\alpha)=0.9\end{cases}
\end{array}
$$

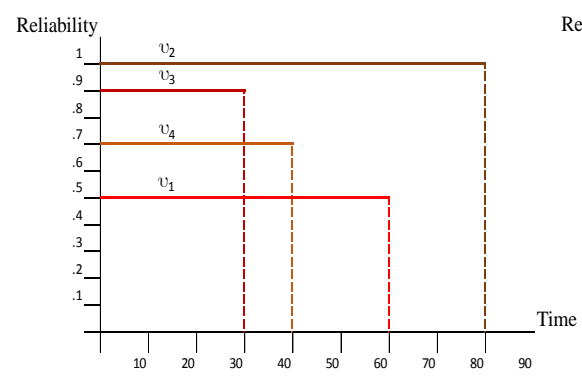

(a) Valuation Functions for each $\tau$-argument belong to $\mathbb{A}$

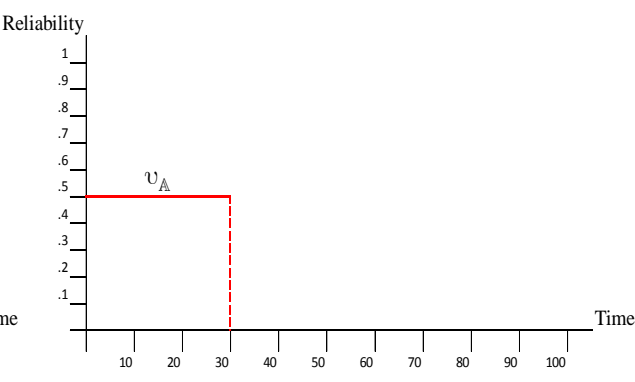

(b) Valuation Function for $\mathbb{A}$

Figure 8: Valuation function associated with $\mathbb{A}$

The set of $\tau^{*}$-structures obtained from the set of $\tau^{*}$-arguments is defined as follows (see Fig. 9):

- $\left(\mathbb{A}, \mathcal{T}_{\mathbb{A}}, v_{\mathbb{A}}\right)$ where $\mathcal{T}_{\mathbb{A}}=\bigcap_{i=1}^{n} \mathcal{T}_{A_{i}}=\{[0-30]\}$ and its corresponding valuation function $v_{\mathbb{A}}=\min \left\{v_{A_{i}}(t) \mid 1 \leq i \leq n, t \in \mathcal{T}_{\mathbb{A}}\right\}$, the process for obtaining $v_{\mathbb{A}}$ is depicted in Fig. 8.

- $\left(\mathbb{B}, \mathcal{T}_{\mathbb{B}}, v_{\mathbb{B}}\right)$ where $\mathcal{T}_{\mathbb{B}}=\bigcap_{i=1}^{n} \mathcal{T}_{B_{i}}=\{[10-50]\}$ and its corresponding valuation function $v_{\mathbb{B}}=\min \left\{v_{B_{i}}(t) \mid 1 \leq i \leq n\right\}$ is depicted in Fig. 10.

- $\left(\mathbb{C}, \mathcal{T}_{\mathbb{C}}, v_{\mathbb{C}}\right)$ where $\mathcal{T}_{\mathbb{C}}=\bigcap_{i=1}^{n} \mathcal{T}_{C_{i}}=\{[0-60]\}$ and its corresponding valuation function $v_{\mathbb{C}}=\min \left\{v_{C_{i}}(t) \mid 1 \leq i \leq n\right\}$ is depicted in Fig. 10.

Note that, in this example the reliability function of the $\tau^{*}$-structure $\left(\mathbb{B}, \mathcal{T}_{\mathbb{B}}, v_{\mathbb{B}}\right)$ and its substructure $\left(\mathbb{B}^{\prime}, \mathcal{T}_{\mathbb{B}^{\prime}}, v_{\mathbb{B}^{\prime}}\right)$ are the same, i.e., $\min \left\{v_{B_{i}}(t) \mid 1 \leq i \leq n\right\}$ for every time point $t$, where $v_{B_{i}}(t)$ is $v_{9}(t)$, and $v_{10}(t)$ coincides with $v_{9}(t)$, which is the reliability function of $\left(\mathbb{B}^{\prime}, \mathcal{T}_{\mathbb{B}^{\prime}}, v_{\mathbb{B}^{\prime}}\right)$.

Atts $=\left\{\left(\left(\mathbb{A}, \mathcal{T}_{\mathbb{A}}, v_{\mathbb{A}}\right),\left(\mathbb{B}, \mathcal{T}_{\mathbb{B}}, v_{\mathbb{B}}\right)\right),\left(\left(\mathbb{B}, \mathcal{T}_{\mathbb{B}}, v_{\mathbb{B}}\right),\left(\mathbb{C}, \mathcal{T}_{\mathbb{C}}, v_{\mathbb{C}}\right)\right)\right\}$ 


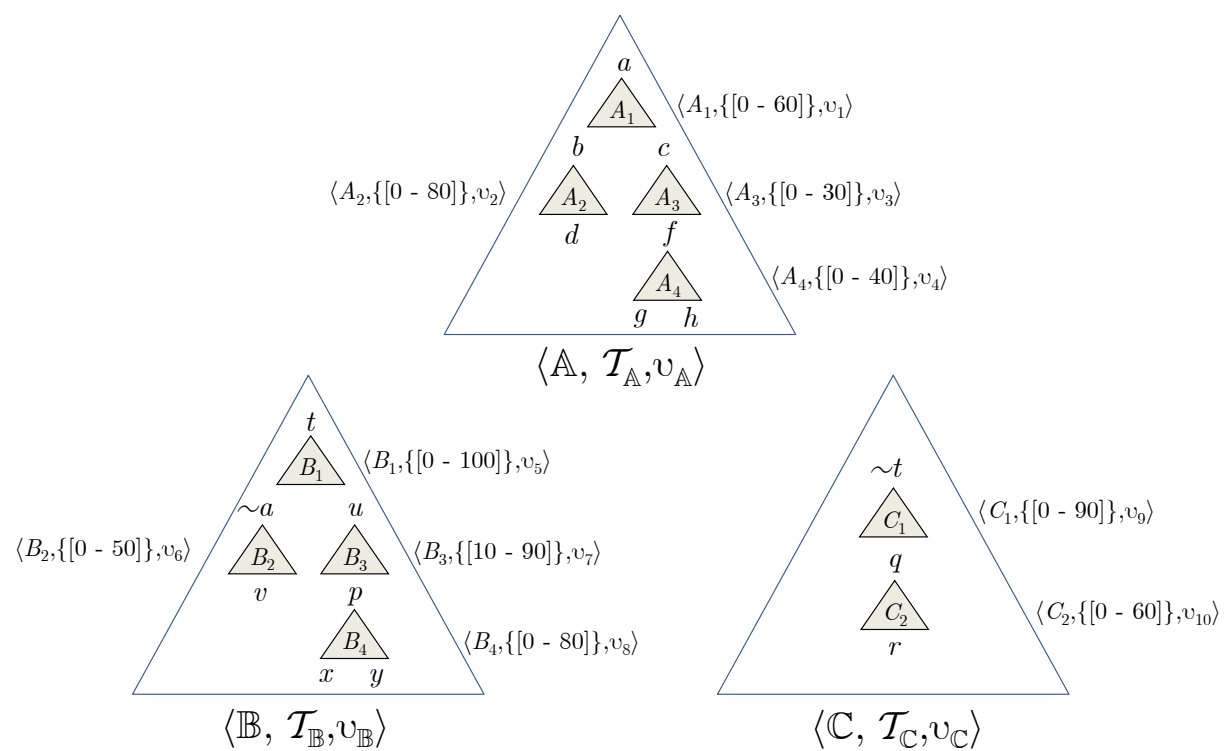

Figure 9: Instance of an $E-T A F^{*}$ (corresponding to Example 8)

$\operatorname{AttAv}\left(\left(\left(\mathbb{A}, \mathcal{T}_{\mathbb{A}}, v_{\mathbb{A}}\right),\left(\mathbb{B}^{\prime}, \mathcal{T}_{\mathbb{B}^{\prime}}, v_{\mathbb{B}^{\prime}}\right)\right)\right)=\left\{\alpha \in \mathbb{R} \mid \alpha \in\{[0-30]\} \quad\right.$ and $\left.v_{\mathbb{B}}(\alpha) \geq v_{\mathbb{A}}(\alpha)\right\}=$

$$
=\{[15-30]\}
$$

$\operatorname{AttAv}\left(\left(\left(\mathbb{B}, \mathcal{T}_{\mathbb{B}}, v_{\mathbb{B}}\right),\left(\mathbb{C}, \mathcal{T}_{\mathbb{C}}, v_{\mathbb{C}}\right),\right)\right)=\left\{\alpha \in \mathbb{R} \mid \alpha \in\{[10-50]\} \quad\right.$ and $\left.v_{\mathbb{B}^{\prime}}(\alpha) \geq v_{\mathbb{C}}(\alpha)\right\}=$

$$
=\{[20-50]\}
$$

This framework coincides with the E-TAF presented in example 4, Fig. 4, for which argument acceptability was already analyzed.

\section{Related Work}

As discussed in the introduction, reasoning about time is a major concern in commonsense reasoning, being also a valuable feature when modeling argumentation capabilities for intelligent agents [7]. There have been also recent advances in modeling time in argumentation frameworks (e.g. [23, 33]). However, to the best of our knowledge, there exists no other abstract argumentation approach for reasoning jointly with time and valuation factors as the one presented in this paper, combining features of $T A F$ and SAF in a single, unified framework.

Recent research has led to Temporal Argumentation Frameworks ( $T A F)$ that extend Dung's $A F$ by considering the temporal availability of arguments $[22$, 23]. In $T A F$, arguments are valid only during specific time intervals (called 


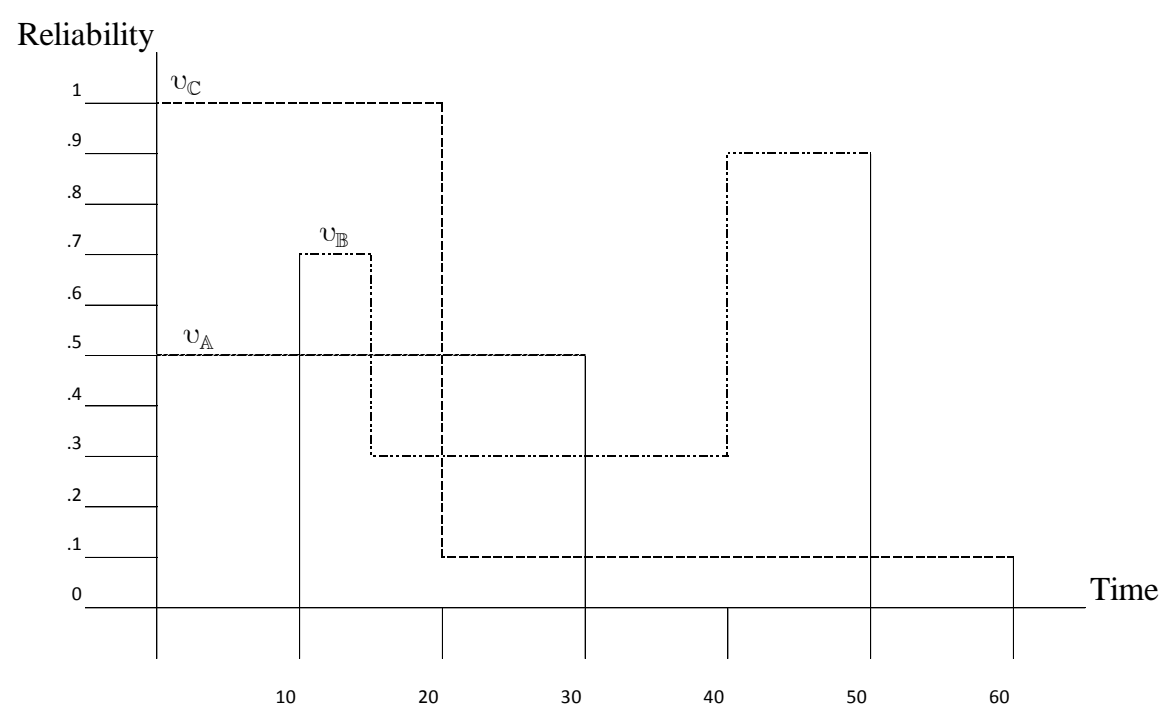

Figure 10: Representation of the valuation functions $v_{\mathbb{A}}, v_{\mathbb{B}}, v_{\mathbb{C}}$

availability intervals). Thus, when identifying the set of acceptable arguments the outcome associated with a TAF may vary in time. The TAF framework allows to model so-called "intermittent arguments", useful in the context of argumentation dynamics. Even though arguments in $T A F$ are associated with availability intervals, in contrast with our approach their attacks are assumed to be static and permanent in time.

In [46], an argumentative approach to reasoning about the trustworthiness of information sources is presented. In contrast with our approach, time is not considered explicitly, and meta-argumentation [15] (which allows Dung's AF to reason about itself) is used to model trust. In contrast, our approach considers valuation functions whose outcomes are based on time intervals. In [37, 34], Prakken \& Modgil present a very rich formalization for adding structure to abstract argumentation. His research has some parallels with the underlying notions in SAF, although it is much more encompassing than ours (considering argument schemes, rationality postulates, etc.). In contrast, our main motivation for structuring arguments was to empower the expressivity of our approach when dealing with valuation.

Associating special constraints or additional information with attacks (as done in this research work) is an idea which has also been explored in other settings. In [47], Villata et al. explore a conceptualization of abstract argumentation in terms of successful and unsuccessful attacks, such that arguments are accepted when there are no successful attacks on them. They characterize the relation between attack semantics and Dung's approach, defining as well a recursive algorithm for attack semantics using attack labeling. More recently [27], 
applications of argumentation to model social networks have come to rely on votes on attacks in order to determine the status of the arguments involved. In our approach, attacks are labeled with time intervals, which is indeed a distinctive feature in comparison with other approaches. It must be noted that the use of time as done in our approach could also be consistently added to the different extensions of Dung's discussed before.

In recent research, assigning valuation to arguments has also resulted in other approaches in which probabilities are used, as the one presented in [31]. This is a powerful probabilistic approach to argumentation, where logical arguments can be qualified by the probability that the premises are true, and the resulting argument graphs can be instantiated by probabilistic logical arguments. Extensions of the argument graph can be qualified by the probability of the logical arguments, and inconsistencies can arise in the probability assignments from multiple sources. In contrast with this approach, in our analysis we do not rely on probabilities, but rather on numerical values propagated within an abstract structured argumentation framework. Additionally, the work in [31] does not consider time as part of the resulting formalization.

Two important approaches that share elements of our research appear in Barringer et al. [9] and [10]. In the first one [9], they studied the relationships of support and attack between arguments through a numerical argumentation network, where they considered both the strength of the arguments, as well as the strength that carry the attack and support between them. This work puts close attention to the relations of support and attack between arguments, and to the treatment of cycles in an argumentative network. In addition, they offered different motivations for modeling domains in which the strengths can be time-dependent, presenting a brief explanation of how to deal with this issue in a numerical argumentation network. In [10], the authors presented a temporal argumentation approach, where they extend the traditional Dung's networks using temporal and modal language formulas to represent the structure of arguments. First, they introduced the concept of usability of arguments defined as a function that determines if an argument is usable or not in a given context, and this status may be changed over time depending on the context dynamics. They also improved the representational capability of the formalism using the ability of modal logic to represent accessibility between different argumentative networks; in this way, the modal operator is treated as a fibring operator to obtain a result for another argumentation network context, and then apply it to the local argumentation network context. As it was mentioned, these represent two research lines that have points in common with our work; however, our motivation is to present a less abstract framework through the structured argumentation frameworks, providing in this way representational advantages. These additional capabilities will open new research lines such as $(i)$ choosing a concrete structured argumentation system to instantiate the abstract structured argumentation such as [16, 37, 13, 29] (see [32] for a set of tutorials); (ii) studying how the aggregation (accrual) of the arguments that support the same conclusion can improve the semantics of argumentation systems, analyzing as well alternative semantics for the underlying argumentation systems; and, (iii) 
finding new ways of taking in consideration the additional meta-information carried by the argumental structures formalized using SAF.

\section{Conclusions and Future Work}

Dung's abstract argumentation framework [25] has been shown useful for developing several extensions which were applied in different contexts (e.g., $[18,17,21,4]$, among many others). As discussed, this paper presents a novel approach in which we integrate features from two separate directions in argumentation. On the one hand, we consider time as a distinctive element, and provide the mechanisms to associate time intervals to attacks. On the other hand, we considered the structure of arguments as a way of abstracting away the structural parts of arguments and their interrelationships (subargument, conflict, etc.). Consequently, we first characterized $E-T A F$, an extension of $T A F$ considering time intervals associated with attacks. Then, based on the notions characterizing SAF, we added structure to $E$-TAF to formalize the notion of valuation for an argument varying on time. The resulting framework $E-T A F^{*}$ incorporates the ability of representing temporal availability and valuation factors associated with the arguments from which the argumental structures are built. This information is then propagated to the level of argumental structures using it to define temporal availability of attacks.

As future work we will develop an implementation of $E-T A F^{*}$ by using the existing DeLP system [29] as a basis. ${ }^{3}$ The resulting implementation will be applied to different domains that require modeling agents associated with a valuation factor varying over time. We are also interested in analyzing the salient features of our formalization in the context of other argumentation frameworks, such as the ASPIC+ framework [37], where rationality postulates for argumentation [20] are explicitly considered. Research in this direction is being pursued.

\section{Appendix A. Proofs}

Proposition 4 Let $\Theta=\langle A R, A t t s, A r g A v, A t t A v\rangle$ be an $E$-TAF, then:

(1) There exist always an at-grounded extension.

(2) An at-preferred extension is also an at-complete extension.

(3) An at-stable extension is also an at-preferred extension.

(4) The at-grounded extension is a t-subset of all at-preferred and at-stable extensions.

\section{Proof.}

(1) From the definition of the characteristic function, at least the empty set is an at-grounded extension.

\footnotetext{
${ }^{3}$ See http://lidia.cs.uns.edu.ar/delp
} 
(2) Results from the definition of at-preferred extension and Proposition 3.

(3) An at-stable extension is the collection of t-profiles that defeat all the tprofiles that are outside the extension; for this reason, this collection of t-profiles must be $\subseteq_{t}$-maximal at-admissible, which is the condition of an at-preferred extension.

(4) The at-grounded extension is the $\subseteq$-minimal complete collection of t-profiles, since an at-preferred extension is equivalently defined as a $\subseteq$-maximal complete collection of t-profiles, it is clear that the at-grounded extension should be included in any at-preferred extension. In addition, from (3) any at-stable extension also should be an at-preferred extension, then the at-grounded extension should be included in all at-stable extensions.

Lemma 1 Let $\Theta=\langle A R, A t t s, A r g A v, A t t A v\rangle$ be an $E-T A F$ and let $\alpha \in \mathbb{R}^{+}$ representing a point in time. Let $\Theta_{\alpha}^{\prime}=\left\langle A R_{\alpha}^{\prime}, A t t s_{\alpha}^{\prime}\right\rangle$ be a Dung abstract framework obtained from $\Theta$ in the following way: $A R_{\alpha}^{\prime}=\left\{A \in A R \mid \alpha \in \mathcal{T}_{A}\right\}$ and Atts $_{\alpha}^{\prime}=\left\{(A, B) \in\right.$ Atts $\left.\mid \alpha \in \mathcal{T}_{(A, B)}\right\}$. Let $E$ a collection of t-profiles in $\Theta$, and $E_{\alpha}^{\prime}=\left\{X \mid\left(X, \mathcal{T}_{(X \mid E)}\right) \in E\right.$ and $\left.\alpha \in \mathcal{T}_{(X \mid E)}\right\}$ (thus, $E_{\alpha}^{\prime} \subseteq A R_{\alpha}^{\prime}$ ). It holds that, if $E$ is an at-preferred extension (respectively an at-stable extension or an at-grounded extension) w.r.t. $\Theta$, then $E_{\alpha}^{\prime}$ is a preferred extension (respectively a stable extension or a grounded extension) w.r.t. $\Theta_{\alpha}^{\prime}$.

PROOF. We will separate the proof in the three parts of the lemma corresponding to each of the three semantics. First, we will prove the general property of conflict-freeness that any extension corresponding to any semantics should satisfy:

If $E$ is an extension w.r.t. $\Theta$, then $E_{\alpha}^{\prime}$ is should be conflict-free w.r.t. $\Theta_{\alpha}^{\prime}$.

Let us assume that $E_{\alpha}^{\prime}$ is not a conflict-free set of arguments. In that case, there should exist two arguments $X, Y \in E_{\alpha}^{\prime}$ such that $(X, Y) \in \operatorname{Atts}_{\alpha}^{\prime}$. From the definition of $E_{\alpha}^{\prime}$, we know that there are $\left(X, \mathcal{T}_{(X \mid E)}\right),\left(Y, \mathcal{T}_{(Y \mid E)}\right) \in E$, such that $\alpha \in\left(X, \mathcal{T}_{(X \mid E)}\right) \cap\left(Y, \mathcal{T}_{(Y \mid E)}\right)$, and $(X, Y) \in$ Atts where $\alpha \in \mathcal{T}_{(X, Y)}$. Consequently, $E$ is not an at-conflict-free set contradicting our initial assumption that $E$ is extension, and this contradiction comes from assuming that $E_{\alpha}^{\prime}$ is not a conflict-free set.

We will now proceed under the assumption that $E_{\alpha}^{\prime}$ is a conflict-free set for the three semantics mentioned.

a) If $E$ is an at-preferred extension w.r.t. $\Theta$, then $E_{\alpha}^{\prime}$ is a preferred extension w.r.t. $\Theta_{\alpha}^{\prime}$.

Let $E$ be an at-preferred extension for $\Theta$, and let $E_{\alpha}^{\prime}$ be a set of arguments such that $E_{\alpha}^{\prime}=\left\{X \mid\left(X, \mathcal{T}_{(X \mid E)}\right) \in E\right.$ and $\left.\alpha \in \mathcal{T}_{(X \mid E)}\right\}$, and let us assume that $E_{\alpha}^{\prime}$ is not a preferred extension of $\Theta_{\alpha}^{\prime}$. For this to be the case, knowing $E_{\alpha}^{\prime}$ is conflict-free, at least one of the two conditions required for preferred semantics should fail, namely: 
i) $E_{\alpha}^{\prime}$ should be an admissible set. Let us assume that $E_{\alpha}^{\prime}$ does not satisfy that condition. In this case, there should exist two arguments $X, Y \in$ $A R_{\alpha}^{\prime}$, such that $Y \notin E_{\alpha}^{\prime}, X \in E_{\alpha}^{\prime},(Y, X) \in A t t s_{\alpha}^{\prime}$ and there should not exist an argument $Z \in A R_{\alpha}^{\prime}$ verifying that $Z \in E_{\alpha}^{\prime}$ and $(Z, Y) \in A_{t t s}^{\prime}$. From the definition of $E_{\alpha}^{\prime}$, we know that there is a t-profile $\left(X, \mathcal{T}_{(X \mid E)}\right) \in E$ where $\alpha \in \mathcal{T}_{(X \mid E)}$, a t-profile $\left(Y, \mathcal{T}_{Y}\right)$ such that $(Y, X) \in$ Atts and $\alpha \in$ $\mathcal{T}_{(Y, X)}$, and does not exist a t-profile $\left(Z, \mathcal{T}_{(Z \mid E)}\right) \in E$ such that $(Z, Y) \in$ Atts, and $\alpha \in \mathcal{T}_{(Z, Y)}$ verifies $\left.\mathcal{T}_{(Y, X)} \cap \mathcal{T}_{(Z, Y)}\right) \cap \mathcal{T}_{X} \neq \emptyset$. But $E$ is an at-preferred extension, and therefore it should satisfies at-admissibility (contradiction).

ii) $E_{\alpha}^{\prime}$ should be a $\subseteq$-maximal set. Let us assume that is not, then there exists a set $E_{\alpha}^{\prime \prime}$ such that $E_{\alpha}^{\prime} \subsetneq E_{\alpha}^{\prime \prime}$ and it satisfies conflict-freeness and admissibility. Let $E_{m}=E \cup\left\{(X,\{\alpha\}) \mid X \in E_{\alpha}^{\prime \prime}\right.$ and $\left.X \notin E_{\alpha}^{\prime}\right\}$. Note that $E \subsetneq_{t} E_{m}$ (by construction). Also, $E_{m}$ is at-admissible. Contradiction, since $E$ is an preferred extension and therefore it is the maximal set w.r.t. t-inclusion which is at-admissible.

b) If $E$ is an at-stable extension w.r.t. $\Theta$, then $E_{\alpha}^{\prime}$ is a stable extension w.r.t. $\Theta_{\alpha}^{\prime}$.

Let $E$ be an at-stable extension for $\Theta$, and let $E_{\alpha}^{\prime}$ be a set of arguments such that $E_{\alpha}^{\prime}=\left\{X \mid\left(X, \mathcal{T}_{X}\right) \in E\right.$ and $\left.\alpha \in \mathcal{T}_{X}\right\}$, and let us assume that $E_{\alpha}^{\prime}$ is not a stable extension of $\Theta_{\alpha}^{\prime}$. For this to be the case, knowing $E_{\alpha}^{\prime}$ is conflict-free, the following condition required for stable semantics should fail: $E_{\alpha}^{\prime}$ should attack all arguments that do not belong to it.

Let us assume that the condition fails; then, there exist at least an argument $X \in A R_{\alpha}^{\prime} \backslash E_{\alpha}^{\prime}$ that is not attacked by any argument in $E_{\alpha}^{\prime}$. Consequently, there exists a t-profile $\left(X, \mathcal{T}_{X}\right) \notin E$, where $E=\left\{\left(Y_{i}, \mathcal{T}_{\left(Y_{i} \mid E\right)}\right) \mid 1 \leq i \leq n\right\}$ such that $\alpha \in \mathcal{T}_{X}$ and therefore $\mathcal{T}_{X} \backslash \bigcup_{i=1}^{n} \mathcal{T}_{\left(Y_{i} \mid E\right)} \neq \emptyset$ since it contains at least the time point $\alpha$. But this is not possible since $E$ is an at-stable extension, thus $E$ attacks all the arguments that do not belong to that at-stable extension, in particular this is true for $\left(X, \mathcal{T}_{X}\right)$. This is a contradiction that arises from our assumption that $E_{\alpha}^{\prime}$ does not attack all arguments that are outside of it. Therefore, $E_{\alpha}^{\prime}$ is a stable extension of $\Theta_{\alpha}^{\prime}$.

c) If $E$ is an at-grounded extension w.r.t. $\Theta$, then $E_{\alpha}^{\prime}$ is a grounded extension w.r.t. $\Theta_{\alpha}^{\prime}$.

Let $E$ be an at-grounded extension for $\Theta$, and let $E_{\alpha}^{\prime}$ be a set of arguments such that $E_{\alpha}^{\prime}=\left\{X \mid\left(X, \mathcal{T}_{X}\right) \in E\right.$ and $\left.\alpha \in \mathcal{T}_{X}\right\}$, and let us assume that $E_{\alpha}^{\prime}$ is not a grounded extension of $\Theta_{\alpha}^{\prime}$. For this to be the case, at least one of the conditions required for grounded semantics should fail:

i) $E_{\alpha}^{\prime}$ should be a complete set. Let us assume that is not, then there exists an argument $Z \in A R_{\alpha}^{\prime}$ such that $Z \notin E_{\alpha}^{\prime}$ and $Z$ is defended by $E_{\alpha}^{\prime}$. In that case, two situations are possible: 
$Z$ is not attacked by any argument of $A R_{\alpha}^{\prime}$. Then, there does not exist an argument $Y \in A R_{\alpha}^{\prime}$ such that $(Y, Z) \in A t t s_{\alpha}^{\prime}$. From the definition of $E_{\alpha}^{\prime}$, we can say that there exists a t-profile $\left(Z, \mathcal{T}_{Z}\right) \notin E$ where $\alpha \in \mathcal{T}_{Z}$, and does not exist a t-profile $\left(Y, \mathcal{T}_{Y}\right)$ such that $(Y, Z) \in$ Atts and $\mathcal{T}_{(Y, Z)} \neq \emptyset$ where $\alpha \in \mathcal{T}_{(Y, Z)}$. Then, $\left(Z, \mathcal{T}_{Z}\right)$ is acceptable with respect to $E$ at least in the time point $\alpha$, but $E$ is an at-grounded extension and therefore it satisfies at-completeness (contradiction).

$Z$ is attacked by another argument of $A R_{\alpha}^{\prime}$. Then, there exists an argument $Y \in A R_{\alpha}^{\prime}$ such that $(Y, Z) \in A_{t t s_{\alpha}^{\prime}}$, and exists an argument $X \in E_{\alpha}^{\prime}$ such that $(X, Y) \in A t t s_{\alpha}^{\prime}$. From the definition of $E_{\alpha}^{\prime}$, we can say that there exists a t-profile $\left(Z, \mathcal{T}_{Z}\right) \notin E$ and $\alpha \in \mathcal{T}_{Z}$, a t-profile $\left(Y, \mathcal{T}_{Y}\right) \in A R$ such that $(Y, Z) \in$ Atts where $\alpha \in \mathcal{T}_{(Y, Z)}$, and a t-profile $\left(X, \mathcal{T}_{(X \mid E)}\right) \in E$ such that $(X, Y) \in$ Atts where $\alpha \in \mathcal{T}_{(X, Y)}$ and verifies $\mathcal{T}_{(Y, Z)} \cap \mathcal{T}_{(X, Y)} \cap \mathcal{T}_{Z} \neq \emptyset$ (since $\left(Z, \mathcal{T}_{Z}\right)$ is defended at least in the time point $\alpha$ for a t-profile of $E$ ). Contradiction, since this situation implies that $E$ is not at-complete, and therefore not at-grounded extension.

ii) $E_{\alpha}^{\prime}$ should be a $\subseteq$-minimal set. Let us assume that is not, then there exists a proper subset $E_{\alpha}^{\prime \prime} \subsetneq E_{\alpha}^{\prime}$ such that satisfies conflict-freeness, admissibility and completeness. Let $E_{m}=\left\{\left(X, \mathcal{T}_{(X \mid E)} \backslash\{\alpha\}\right) \mid\left(X, \mathcal{T}_{(X \mid E)}\right) \in E\right.$ and $X \notin$ $\left.E_{\alpha}^{\prime \prime}\right\}$. Note that $E \subseteq_{t} E_{m}$ (for construction). Also, $E_{m}$ is at-complete and at-admissible. Contradiction, since $E$ is a grounded extension and for that is a minimal set w.r.t. t-inclusion which is at-complete and at-admissible.

Proposition 6 Given the $E-T A F^{*} \Psi=\left\langle\operatorname{Str}_{(\operatorname{Args}, \bowtie)}, \asymp, A t t A v, \geq\right\rangle$ and the two $\tau^{*}$-structures $\left(\mathbb{A}, \mathcal{T}_{\mathbb{A}}, v_{\mathbb{A}}\right),\left(\mathbb{B}, \mathcal{T}_{\mathbb{B}}, v_{\mathbb{B}}\right) \in \operatorname{Str}(\operatorname{Args}, \bowtie)$. If the $\tau^{*}$-structure $\left(\mathbb{A}, \mathcal{T}_{\mathbb{A}}, v_{\mathbb{A}}\right)$ defeats $\left(\mathbb{B}^{\prime}, \mathcal{T}_{\mathbb{B}^{\prime}}, v_{\mathbb{B}^{\prime}}\right)$ over the time interval $\mathcal{T}_{\mathbb{A}} \cap \mathcal{T}_{\mathbb{B}^{\prime}}$, where $\left(\mathbb{B}^{\prime}, \mathcal{T}_{\mathbb{B}^{\prime}}, v_{\mathbb{B}^{\prime}}\right)$ is a $\tau^{*}$ substructure of $\left(\mathbb{B}, \mathcal{T}_{\mathbb{B}}, v_{\mathbb{B}}\right)$ then it holds that:

- $\mathcal{T}_{\mathbb{A}}^{P r_{\Psi}} \cap \mathcal{T}_{\mathbb{B}}^{P r_{\Psi}}=\emptyset$,

- $\mathcal{T}_{\mathbb{A}}^{S t_{\Psi}} \cap \mathcal{T}_{\mathbb{B}}^{S t t_{\Psi}}=\emptyset$, and

- $\mathcal{T}_{\mathbb{A}}^{G r_{\Psi}} \cap \mathcal{T}_{\mathbb{B}}^{G r_{\Psi}}=\emptyset$.

Proof. Let $E$ be the at-grounded extension for $\Psi$. Let $\left(\mathbb{A}, \mathcal{T}_{\mathbb{A}}, v_{\mathbb{A}}\right),\left(\mathbb{B}, \mathcal{T}_{\mathbb{B}}, v_{\mathbb{B}}\right)$ be two $\tau^{*}$-structures in $E$. Since $E$ is the at-grounded extension for $\Psi$, then $E$ is a set that it is minimal w.r.t. t-inclusion, at-admissible, and at-complete. For definition of at-admissible $E$ is a at-conflict-free set, then there is no $\tau^{*}$ structures $\left(\mathbb{A}, \mathcal{T}_{\mathbb{A}}, v_{\mathbb{A}}\right)$ and $\left(\mathbb{B}, \mathcal{T}_{\mathbb{B}}, v_{\mathbb{B}}\right) \in E$ such that $\left(\mathbb{A}, \mathcal{T}_{\mathbb{A}}, v_{\mathbb{A}}\right),\left(\mathbb{B}, \mathcal{T}_{\mathbb{B}}, v_{\mathbb{B}}\right) \in \asymp$ and $\mathcal{T}_{\mathbb{A}} \cap \mathcal{T}_{\mathbb{B}} \neq \emptyset$. Therefore, $\mathcal{T}_{\mathbb{A}}^{G r_{\Psi}} \cap \mathcal{T}_{\mathbb{B}}^{G r_{\Psi}}=\emptyset$.

The proof of the result is based on the property that states that the set $E$ is conflict free, meaning there cannot be a conflict between two elements of $E$. This condition is necessary for the three extensions (at-grounded, at-stable and at-preferred), and consequently the proof for the other two extensions is analogous. 
Proposition 7 Let $\Psi$ be an $E$ - TAF $F^{*}$ and $\left(\mathbb{A}, \mathcal{T}_{\mathbb{A}}, v_{\mathbb{A}}\right),\left(\mathbb{A}^{\prime}, \mathcal{T}_{\mathbb{A}^{\prime}}, v_{\mathbb{A}^{\prime}}\right) \in \operatorname{Str}(\operatorname{Args}, \bowtie)$, such that $\left(\mathbb{A}^{\prime}, \mathcal{T}_{\mathbb{A}^{\prime}}, v_{\mathbb{A}^{\prime}}\right)$ is a $\tau^{*}$-substructure of $\left(\mathbb{A}, \mathcal{T}_{\mathbb{A}}, v_{\mathbb{A}}\right)$. Then:

$$
\begin{aligned}
-\mathcal{T}_{\mathbb{A}^{\prime}}^{G r_{\Psi}} & \supseteq \mathcal{T}_{\mathbb{A}}^{G r r_{\Psi}} . \\
-\mathcal{T}_{\mathbb{A}^{\prime}}^{P r_{\Psi}} & \supseteq \mathcal{T}_{\mathbb{A}}^{P r_{\Psi}}, \text { and } \\
-\mathcal{T}_{\mathbb{A}^{\prime}}^{S t_{\Psi}} & \supseteq \mathcal{T}_{\mathbb{A}}^{S t_{\Psi}},
\end{aligned}
$$

Proof. Let $\left(\mathbb{A}^{\prime}, \mathcal{T}_{\mathbb{A}^{\prime}}, v_{\mathbb{A}^{\prime}}\right)$ be a $\tau^{*}$-substructure of $\left(\mathbb{A}, \mathcal{T}_{\mathbb{A}}, v_{\mathbb{A}}\right)$. From the definition of $\tau^{*}$-substructure it holds that $\operatorname{args}\left(\mathbb{A}^{\prime}\right) \subseteq \operatorname{args}(\mathbb{A})$ where $\operatorname{args}\left(\mathbb{A}^{\prime}\right)$ and $\operatorname{args}(\mathbb{A})$ are sets of $\tau^{*}$-arguments. Also, from the definition of $\tau^{*}$-structure it holds that the time interval in which the $\tau^{*}$-structure is available is $\mathcal{T}_{\mathbb{A}}=$ $\bigcap_{i=1}^{n} \mathcal{T}_{A_{i}}$ for each $\tau^{*}$-argument $\left\langle A_{i}, \mathcal{T}_{i}, v_{i}\right\rangle \in \operatorname{args}(\mathbb{A})$.

The time interval corresponding to a $\tau^{*}$-structure in the at-grounded extension is obtained considering the intersection of all the time intervals of the $\tau^{*}$-argument that are in it.

The time interval in which a $\tau^{*}$-structure belongs to the at-grounded extension is obtained considering the intersection of all the time intervals of each $\tau^{*}$-argument belong to the at-grounded extension. Formally,

$$
\mathcal{T}_{\mathbb{A}}^{G r_{\Psi}}=\bigcap_{i=1}^{n} \mathcal{T}_{A_{i}}^{G r_{\Psi}}
$$

Since $\operatorname{args}\left(\mathbb{A}^{\prime}\right) \subseteq \operatorname{args}(\mathbb{A})$, then

$$
\mathcal{T}_{\mathbb{A}}^{G r_{\Psi}}=\bigcap_{A_{i} \in \operatorname{args}(\mathbb{A})} \mathcal{T}_{A_{i}}^{G r_{\Psi}} \subseteq \bigcap_{A_{i} \in \operatorname{args}\left(\mathbb{A}^{\prime}\right)} \mathcal{T}_{A_{i}}^{G r_{\Psi}}=\mathcal{T}_{\mathbb{A}^{\prime}}^{G r_{\Psi}}
$$

since $\operatorname{args}\left(\mathbb{A}^{\prime}\right) \subseteq \operatorname{args}(\mathbb{A})$.

The proofs for the other two semantics are analogous.

\section{References}

[1] Allen, J. F., 1983. Maintaining knowledge about temporal intervals. Commun. ACM 26 (11), 832-843.

[2] Amgoud, L., Cayrol, C., 1998. On the acceptability of arguments in preference-based argumentation. In: Cooper, G., Moral, S. (Eds.), UAI. Morgan Kaufmann, USA, pp. 1-7.

[3] Amgoud, L., Cayrol, C., 2002. A reasoning model based on the production of acceptable arguments. Ann. Math. Artif. Intell. 34 (1-3), 197-215.

[4] Amgoud, L., Devred, C., 2011. Argumentation frameworks as constraint satisfaction problems. In: Benferhat, S., Grant, J. (Eds.), SUM. Vol. 6929 of Lecture Notes in Computer Science. Springer, pp. 110-122. 
[5] Amgoud, L., Prade, H., 2009. Using arguments for making and explaining decisions. Artificial Intelligence 173 (3-4), 413-436.

[6] Augusto, J. C., Simari, G. R., 1999. A temporal argumentative system. AI Commun. 12 (4), 237-257.

[7] Augusto, J. C., Simari, G. R., 2001. Temporal defeasible reasoning. Knowledge and Information Systems 3 (3), 287-318.

[8] Baroni, P., Cerutti, F., Giacomin, M., Simari, G. R. (Eds.), 2010. Computational Models of Argument: Proc. of COMMA 2010. Vol. 216 of Frontiers in Artificial Intelligence and Applications. IOS Press, Italy.

[9] Barringer, H., Gabbay, D., Woods, J., 2012. Temporal, numerical and metalevel dynamics in argumentation networks. Argument \& Computation 3 (23), 143-202.

[10] Barringer, H., Gabbay, D. M., Woods, J., 2012. Modal and temporal argumentation networks. Argument \& Computation 3 (2-3), 203-227.

[11] Bench-Capon, T. J. M., Araszkiewicz, M., Ashley, K. D., Atkinson, K., Bex, F., Borges, F., Bourcier, D., Bourgine, P., Conrad, J. G., Francesconi, E., Gordon, T. F., Governatori, G., Leidner, J. L., Lewis, D. D., Loui, R. P., McCarty, L. T., Prakken, H., Schilder, F., Schweighofer, E., Thompson, P., Tyrrell, A., Verheij, B., Walton, D. N., Wyner, A. Z., 2012. A history of $\mathrm{AI}$ and Law in 50 papers: 25 years of the International Conference on AI and Law. Artif. Intell. Law 20 (3), 215-319.

[12] Bench-Capon, T. J. M., Dunne, P. E., 2007. Argumentation in Artificial Intelligence. Artif. Intell. 171 (10-15), 619-641.

[13] Besnard, P., Hunter, A., 2001. A logic-based theory of deductive arguments. Artif. Intell. 128 (1-2), 203-235.

[14] Besnard, P., Hunter, A., 2008. Elements of argumentation. Vol. 47. MIT press Cambridge.

[15] Boella, G., van der Torre, L., Villata, S., 2009. On the acceptability of meta-arguments. In: IAT. pp. 259-262.

[16] Bondarenko, A., Dung, P., Kowalski, R., Toni, F., 1997. An abstract, argumentation-theoretic approach to default reasoning. Artificial Intelligence 93 (1-2), 63-101.

[17] Brewka, G., Dunne, P. E., Woltran, S., 2011. Relating the semantics of abstract dialectical frameworks and standard afs. In: [48], pp. 780-785.

[18] Brewka, G., Woltran, S., 2010. Abstract dialectical frameworks. In: Lin, F., Sattler, U., Truszczynski, M. (Eds.), KR. AAAI Press. 
[19] Budán, M. C., Gómez Lucero, M. J., Chesñevar, C. I., Simari, G. R., 2012. Modelling time and reliability in structured argumentation frameworks. In: Brewka, G., Eiter, T., McIlraith, S. A. (Eds.), KR. AAAI Press, Italy.

[20] Caminada, M., Amgoud, L., 2007. On the evaluation of argumentation formalisms. Artif. Intell. 171 (5-6), 286-310.

[21] Caminada, M., Pigozzi, G., 2011. On judgment aggregation in abstract argumentation. Autonomous Agents and Multi-Agent Systems 22 (1), 64102.

[22] Cobo, M. L., Martínez, D. C., Simari, G. R., 2010. On admissibility in timed abstract argumentation frameworks. In: Coelho, H., Studer, R., Wooldridge, M. (Eds.), ECAI. Vol. 215 of Frontiers in Artificial Intelligence and Applications. IOS Press, pp. 1007-1008.

[23] Cobo, M. L., Martínez, D. C., Simari, G. R., 2011. Acceptability in timed frameworks with intermittent arguments. In: Iliadis, L. S., Maglogiannis, I., Papadopoulos, H. (Eds.), EANN/AIAI (2). Vol. 364 of IFIP Publications. Springer, pp. 202-211.

[24] Dung, P. M., 1993. On the acceptability of arguments and its fundamental role in nonmonotonic reasoning and logic programming. In: Bajcsy, R. (Ed.), IJCAI. Morgan Kaufmann, pp. 852-859.

[25] Dung, P. M., 1995. On the acceptability of arguments and its fundamental role in nonmonotonic reasoning and logic programming and $n$-person games. Artificial Intelligence 77, 321-357.

[26] Dung, P. M., Kowalski, R. A., Toni, F., 2009. Assumption-based argumentation. In: Rahwan, I., Simari, G. R. (Eds.), Argumentation in Artificial Intelligence. Springer, pp. 198-218.

[27] Egilmez, S., Leite, J., Martins, J., 2013. Extending social abstract argumentation with votes on attacks. In: Prof. of TAFA 2013, IJCAI 2013, Beijing, China. p. (in press).

[28] Fisher, M., 2008. Temporal representation and reasoning. In: van Harmelen, F., Lifschitz, V., Porter, B. (Eds.), Handbook of Knowledge Representation. Foundations of Artificial Intelligence. Elsevier, Ch. 12, pp. 513-550.

[29] García, A. J., Simari, G. R., 2004. Defeasible logic programming: An argumentative approach. Theory Practice of Logic Programming 4 (1), 95-138.

[30] Gordon, T. F., 1993. The pleadings game. Artif. Intell. Law 2 (4), 239-292.

[31] Hunter, A., 2013. A probabilistic approach to modelling uncertain logical arguments. International Journal of Approximate Reasoning 54 (1), 47 81. 
[32] Hunter, A., 2014. Special Issue: Tutorials on Structured Argumentation. Argument \& Computation, Volume 5, Issue 1, (A. Hunter editor).

[33] Mann, N., Hunter, A., 2008. Argumentation using temporal knowledge. In: Proceedings of the 2nd International Conf. on Computational Models of Argument (COMMA 2008). pp. 204-215.

[34] Modgil, S., Prakken, H., 2010. Reasoning about preferences in structured extended argumentation frameworks. In: [8], pp. 347-358.

[35] Modgil, S., Toni, F., Bex, F., Bratko, I., Chesñevar, C. I., Dvořák, W., Falappa, M. A., Fan, X., Gaggl, S. A., García, A. J., et al., 2013. The added value of argumentation. In: Agreement Technologies. Springer, pp. $357-403$.

[36] Pasquier, P., Hollands, R., Rahwan, I., Dignum, F., Sonenberg, L., 2011. An empirical study of interest-based negotiation. Autonomous Agents and Multi-Agent Systems 22 (2), 249-288.

[37] Prakken, H., 2010. An abstract framework for argumentation with structured arguments. Argument and Computation 1, 93-124.

[38] Rahwan, I., Ramchurn, S. D., Jennings, N. R., Mcburney, P., Parsons, S., Sonenberg, L., December 2003. Argumentation-based negotiation. Knowl. Eng. Rev. 18, 343-375.

[39] Rahwan, I., Simari, G. R., 2009. Argumentation in artificial intelligence. Springer.

[40] Rahwan, I., Simari, G. R., 2009. Argumentation in Artificial Intelligence. Springer Verlag.

[41] Rotstein, N. D., Moguillansky, M. O., García, A. J., Simari, G. R., 2010. A dynamic argumentation framework. In: [8], pp. 427-438.

[42] Simari, G. R., Loui, R. P., 1992. A Mathematical Treatment of Defeasible Reasoning and its Implementation. Artificial Intelligence 53, 125-157.

[43] Stolzenburg, F., García, A. J., Chesñevar, Carlos, I., Simari, G. R., 2003. Computing Generalized Specificity. J. of N.Classical Logics 13 (1), 87-113.

[44] van der Weide, T. L., Dignum, F., Meyer, J.-J. C., Prakken, H., Vreeswijk, G., 2011. Multi-criteria argument selection in persuasion dialogues. In: Sonenberg, L., Stone, P., Tumer, K., Yolum, P. (Eds.), AAMAS. IFAAMAS, pp. 921-928.

[45] Vila, L., 1994. A survey on temporal reasoning in artificial intelligence. AI Communications 7 (1), 4-28. 
[46] Villata, S., Boella, G., Gabbay, D. M., van der Torre, L., 2011. Arguing about the trustworthiness of the information sources. In: Proc. of ECSQARU Intl. Conf. pp. 74-85.

[47] Villata, S., Boella, G., van der Torre, L., 2011. Attack semantics for abstract argumentation. In: [48], pp. 406-413.

[48] Walsh, T. (Ed.), 2011. IJCAI 2011, Proceedings of the 22nd International Joint Conference on Artificial Intelligence, Barcelona, Catalonia, Spain, July 16-22, 2011. IJCAI/AAAI. 